\title{
Does the January Effect Still Exists?
}

\author{
Gerardo "Gerry" Alfonso Perez" \\ ${ }^{1}$ University of Cambridge, UK \\ Correspondence: Gerardo “Gerry” Alfonso Perez, University of Cambridge, UK.
}

Received: August 24, 2017

Accepted: October 2, 2017

Online Published: December 3, 2017

doi:10.5430/ijfr.v9n1p50

URL: https://doi.org/10.5430/ijfr.v9n1p50

\begin{abstract}
The issue of the January Effect has attracted a lot of interest by both practitioners and researchers. The idea that stock returns in January are statistically bigger than in other months was first presented several decades ago. This study analyzes the issue of the January effect in a systematic and global way of studying the performance of 106 indexes in 86 countries and jurisdictions. It was observed that while this effect can still be appreciated in some markets it would appear that it is decreasing globally over time. It was also found that there appears to be an Inverted January Effect in several markets with the returns in January being lower than the returns in some other months. This analysis was performed with nonparametric tests. The hypothesis that the returns of the indexes do not follow in general a normal distribution was also confirmed with several tests.
\end{abstract}

Keywords: January effect, market anomalies, stock returns

\section{Introduction}

There are a large number of market abnormalities identified both in the academic literature as well as by practitioners. One of these abnormalities is the January effect. The January effect refers to the observation that returns in January appear to be higher than returns in other months. One of the firsts, if not the first, academic article describing the January Effect was (Watchel, 1942). Since then several authors, such as (Haugen, 1988), (Thaler, 1987), (Jones, 1989), (Moller, 2008), (He, 2011), have analyzedthis issue.The existence of a January effect, as many other market abnormalities, has been used as an argument supporting the idea that markets are not completely efficient. The idea behind this approach is that if such market abnormality exist and can be exploited for trading purposes then, at least in principle, it would be possible to outperform the market in a consistent way, which would contradict the market efficiency hypothesis. The scope of this article it is not to study the link between abnormal returns and market efficiencies but to analyze, in a global basis, what markets present this phenomenon.

The January effect has been observed in several countries for some specific time periods such as for instance the U.S. for some decades after World War I (Charles, 1989). The absence of a January effect before World War I was detected in other countries such as Germany (Taufiq, 2016). Interestingly, the results of (Taufiq, 2016) for the U.K. and the U.S. suggest that there was a January Effect in the pre-war period which seems to contradict several articles such as (Schultz, 1985). The majority of the literature available seems to support the hypothesis that there was no such effect for the period before the World War I, particularly in the U.S. Other countries where the January Effect has been detected are Japan (Li, 2015), Jordan, Morocco from 1988 to 2014 (Gharaibeh, 2017), Turkey (Guler, 2013), India (Kaur, 2017) and several countries in Western Europe (Asteriou, 2006) from 1991 to 2003. In some other markets, like Pakistan, an abnormal return in January has been identified (Hashmi, 2014) but the authors mentioned that the effect is small and no profitable strategy can be built after accounting for transaction costs. It is also interesting that the January effect seems to be changing over time with several articles, such as (Gu, 2013), (Mehdian, 2002), (Patel, 2016) pointing to a declining January effect in the U.S. market. In these articles the authors observed a decline in the effect in the U.S. market starting in the late eighties. The performance in January has been even treated as a precursor of the performance for the rest of the year (Cooper, 2006). There are also abundant articles defending the idea that there is no January effect in some markets such as New Zealand (Li, 2010), India (Mehta, 2009), (Pandeu, 2016) or Indonesia (Simbolon, 2015). The January Effect has been studied not only in equities but also in fixed income investments. For instance, (Starks, 2006) detected the presence of a January Effect on closed end municipal bond funds. Interestingly the authors detected the presence of a January effect on the funds but not on the bonds constituting these funds. The authors attribute these results to tax harvesting. 
The idea that stock returns in one month could be higher than in another month could be supported by cyclical factors and human behavior. In principle there is no clear reason supporting the idea that stock performance should be stronger in January that in other months. Some of the frequently mentioned explanations are:

\subsection{Tax rationale}

One of the frequently mentioned potential explanations for this event in the U.S. is the federal income tax effect (Jones, 1989) with the existing academic literature supporting that there was no January effect before the introduction of federal income tax. It should be noted that there is less of an agreement of the existence of the January effect for some of the years just after the introduction of federal income taxes. Related to tax issues, other authors have found that there has been no obvious impact in the January Effect by some large tax reforms such as for instance the Reform Act in 1986 (Haug, 2006). Tax reasons (Honghui, 2004) are among the most frequently cited explanations for the January effect.The idea behind a tax argument is rather simple. Investors in order to minimize their annual tax bill sell some of the losing positions before year end to increase their losses. This is commonly known as tax harvesting. A byproduct of this tax harvesting is a larger cash pool in January that it is then reinvested pushing prices up. It should also be noted thecaveat mentioned by (Taufiq, 2016) that the German case cannot be used for analyzing the impact of taxes in the January effect as there were no applicable capital gain taxes in Germany during that period (Taufiq, 2016). The tax argument is however not universally accepted with articles such as (Gu, 2005) providing some empirical evidence against it in the Chinese market.

\subsection{Psychological Rationale}

Psychological factors are also frequently used in an attempt to explain the reasons behind the January effect (Anderson, 2007). Some authors, such as (Ciccone, 2011), have mentioned that the new year is a period of renewed optimism and that such optimism likely spreads also to the stock market. This is clearly an explanation not related to market fundamentals but to human behavior. (Ciccone, 2011) does provide some quantitative data. For instance, the authors mentioned that the University of Michigan Consumer Confidence Index tends to peak in January, which is used in this article as a proxy for investors optimism.

\subsection{Window Dressing}

Window dressing is one of the most popular explanations behind this effect (Haugen, 1988), (Klock, 2014). The idea is that fund managers will try to make their portfolios look as good as possible by the end of the year. In order to do that they will sell losing stocks during the end of the year, keeping those that have performed strongly. The funds that were obtained from those stock are then reinvested in January in more speculative stocks in an attempt to obtain strong performances. This inflow of funds in January will push prices up, causing the returns in the month of January to increase.

\subsection{Gifts}

Another explanation of the January effect, postulated by (Gamble, 1993), suggests that the January effect is caused by gifts, typically to younger investors, during the end of year and Christmas festivities. The idea is that some of the cash gifts are invested in the stock market. More specifically, the author suggests that older investors tend to own stable, well capitalized companies and sell a fraction of those holdings at the end of the year to free up funds for gifts. Always according to the author, these stable, large companies are only moderately impacted by this selling. Younger investors tend to more speculative investors, favoring smaller companies. Purchases of those names are likely to push the prices up given their comparatively poor liquidity, driving the market up.

Regardless of what is the real reason behind this effect it seems reasonable to try to determine for which markets there is empirical evidence of such effect actually happening that is the main objective of this article. In an attempt to answer that question a very large data base of stock returns across many countries and jurisdictions was analyzed. The steps followed for this analysis are presented in the next section.

\section{Methodology}

\subsection{Hypothesis}

The null hypothesis in this article is that the returns in the month of January are not statistically different from the returns on any other month of the year. This analysis is performed on a global basis including a large amount of countries and jurisdictions. It was not assumed that the returns of the indexes follow a normal distribution. There is ample literature supporting the argument that stocks returns are not normally distributed. Nevertheless, several tests were carried out to confirm such assumption. 


\subsection{Data}

The data are composed of monthly closing values of 106 indexes covering 86 countries and jurisdictions. It includes indexes representing supranational entities such as Europe or the GCC as well as special administrative areas such as Hong Kong in China. According to data from Bloomberg the combined market capitalization of those 84 countries and jurisdictions accounted for approximately $92.3 \%$ of the global market capitalization as of July 2017 . There is no double counting, with the estimate excluding the market capitalization of supranational indexes such as those covering Europe or the GCC.

The length of the time series varies from country to country and from index to index. The Dow Jones index for instance has a much longer time series than some of the emerging markets indexes. The analysis was performed using the entire data set for each index as well as using, from comparability purposes, only the last 15 years of values as of end of June 2017. For consistency in all the cases the same numbers of data points per month were used. All the data were obtained from Bloomberg. Monthly returns were obtained using monthly closing prices and formula [1]. The data was then grouped by month (from January to December).

$$
\text { Monthly Return }_{T}=\frac{\text { Closing price }_{T}}{\text { Closing price }_{T-1}}-1
$$

\subsection{Procedure}

In a preliminary test the hypothesis that the index returns are normally distributed were checked with a Lillie test and an Anderson Darling test for each index for every month. As expected, for most cases the hypothesis that the index returns are normally distributed was rejected at a 5\% confidence level. The results of the Anderson-Darling and the Lillie tests for every month for every index can be found in Appendix 2 and Appendix 3. The null hypothesis of the Anderson Darling test is that the data come follow a normal distribution. For the vast majority of the indexes the hypothesis that the monthly returns follow a normal distribution (for all the months of the year) cannot be accepted. According to the Anderson Darling test there were only 4 indexes, out of the 106 analyzed, in which the assumption that the returns follow a normal distribution for all the 12 months of the year cannot be rejected. Those four indexes are the PSI All Share (Portugal), Nigerian Stock Exchange Index (Nigeria), Tunisian Stock Exchange Index (Tunisia) and the S\&P NZX All Index (New Zealand). Using the Lillie test similar results were obtained with no rejection of the null hypothesis of a normal distribution only in 10 out of 106 indexes analyzed at a 5\% significance level. The null hypothesis in the Lillie test is that the underlying data follows a normal distribution. The 10 indexes for which the hypothesis that their returns follow a normal distribution are the S\&P 1500 (U.S.), Colombia Colcap Index (Colombia), Ibex 35 (Spain), PSI All Share All Share Index (Portugal), Oslo All Share Index (Norway), Vienna Stock Exchange Index (Austria), Tunisia Stock Exchange Index (Tunisia), Nigeria Stock Exchange Index (Nigeria), Tadawull All Share Index (Saudi Arabia) and Bloomberg GCC 200 (GCC).Given that for the vast majority of the indexes the monthly returns do not appear to follow normal distribution hence nonparametric tests, such as the Wilcoxon Rank Sum and the Kruskal Wallis tests, were used to compare the returns. These tests do not assume that the data follows a normal distribution. The Wilcoxon test compares the medians of two data sample to determine if they are statistically equal at a certain confidence level. The purpose of the Kruskal Wallis test is determining if two, or more, samples of data come from the same distribution or not at a determined confidence level.

The returns in January were compared with the returns for all the other eleven months of the year using the Wilcoxon test. The results for the Wilcoxon tests for the entire available data set for the data provider can be found in Table 2 . The results obtained using the Kruskal Wallis test can be found in Table 4. Given that the entire data series available for each index are not of the same size it seemed reasonable, for comparability purposes, to do some further analysis using the same data time period for all the indexes. The time period used was 15 years (ending in June 2017). It should be noted that not all the indexes have a time series of monthly returns for 15 years. In fact, of the 106 indexes analyzed 16 did not have data available for the required period. The list of indexes that did not fulfill this requirement can be seen in Table 1.

Table 1. Excluded indexes

\begin{tabular}{lll}
\hline FTSE Italia All Share (Italy) & Tanzania All Sh. Ind (Tanzania) & Dubai Fin. Market Ind. (Dubai) \\
\hline Ljubljana St.Ind (Slovenia) & Nairobi Sec. Exc. All (Kenya) & Chile 65 (Chile) \\
\hline St. Ex. Rep.Srpska (Serbia) & Ghana Composite Ind. (Ghana) & Bloomberg GCC (GCC) \\
\hline MBI 10 (Macedonia) & Kuwait St. Exc. Ind. (Kuwait) & Laos Comp. Ind. (Laos) \\
\hline Cyprus Gen. Exc.Ind (Cyprus) & Bahrain Bourse All (Bahrain) & \\
\hline FTSE JSE Nam. Ind (Namibia) & QE All Share (Qatar) & \\
\hline
\end{tabular}


The same process as before was repeated with these shorter times with a Wilcoxon and a Kruskal Wallis test performed in all of indexes. The results of the Wilcoxon test for this reduced data series can be found on Table 3 while the results of the Kruskal Wallis tests can be found on Table 4.

Table 2. Wilcoxon test comparing January returns with the rest of month (p-values) for the entire time series

\begin{tabular}{|c|c|c|c|c|c|c|c|c|c|c|c|c|}
\hline Index & Location & Feb & Mar & Apr & May & Jun & Jul & Aug & Sept & Oct & Nov & Dec \\
\hline S\&P 500 & U.S. & 0.773 & 0.139 & 0.977 & 0.192 & 0.347 & 0.369 & 0.049 & 0.198 & 0.625 & 0.401 & 0.316 \\
\hline Dow Jones & U.S. & 0.480 & 0.042 & 0.682 & 0.171 & 0.548 & 0.146 & 0.006 & 0.912 & 0.916 & 0.219 & 0.122 \\
\hline Nasdaq Com. & U.S. & 0.393 & 0.461 & 0.615 & 0.186 & 0.984 & 0.577 & 0.885 & 0.48 & 0.671 & 0.794 & 0.848 \\
\hline Nasdaq 100 & U.S. & 0.189 & 0.58 & 0.208 & 0.196 & 0.962 & 0.978 & 0.895 & 0.857 & 0.558 & 0.54 & 0.778 \\
\hline N.Y.S.E.I & U.S. & 0.825 & 0.723 & 0.925 & 0.43 & 0.306 & 0.105 & 0.048 & 0.763 & 1 & 0.143 & 0.117 \\
\hline S\&P 100 & U.S. & 0.773 & 0.139 & 0.977 & 0.192 & 0.347 & 0.369 & 0.049 & 0.198 & 0.625 & 0.401 & 0.316 \\
\hline S\&P 1500 & U.S. & 0.534 & 0.805 & 0.379 & 0.991 & 0.193 & 0.103 & 0.46 & 0.614 & 0.879 & 0.474 & 0.405 \\
\hline Russell 1000 & U.S. & 0.269 & 0.523 & 0.543 & 0.116 & 0.651 & 0.264 & 0.659 & 0.823 & 0.622 & 0.996 & 0.831 \\
\hline Russell 2000 & U.S. & 0.483 & 0.234 & 0.682 & 0.123 & 0.557 & 0.306 & 0.347 & 0.921 & 0.938 & 0.897 & 0.791 \\
\hline Russell 3000 & U.S. & 0.182 & 0.55 & 0.477 & 0.116 & 0.682 & 0.255 & 0.636 & 0.848 & 0.682 & 0.913 & 0.946 \\
\hline S\&P Toronto & Canada & 0.048 & 0.193 & 0.8 & 0.196 & 0.293 & 0.378 & 0.132 & 0.234 & 0.76 & 0.75 & 0.851 \\
\hline S\&P BMV & Mexico & 0.948 & 0.056 & 0.496 & 0.983 & 0.416 & 0.758 & 0.861 & 0.775 & 0.482 & 0.282 & 0.141 \\
\hline Bol.al .Pan. & Panama & 0.048 & 0.193 & 0.8 & 0.196 & 0.293 & 0.378 & 0.132 & 0.234 & 0.76 & 0.75 & 0.851 \\
\hline B.A. .In. & Argentina & 0.901 & 0.852 & 0.78 & 0.144 & 0.721 & 0.576 & 0.106 & 0.256 & 0.828 & 0.208 & 0.27 \\
\hline Ibovespa & Brazil & 0.438 & 0.295 & 0.587 & 0.393 & 0.426 & 0.181 & 0.14 & 0.801 & 0.13 & 0.194 & 0.174 \\
\hline Chile 65 & Chile & 0.977 & 0.977 & 0.84 & 0.751 & 0.237 & 0.175 & 0.997 & 0.126 & 0.26 & 0.126 & 0.089 \\
\hline Caracas In. & Venezuela & 0.843 & 0.75 & 0.598 & 0.598 & 0.725 & 0.792 & 0.328 & 0.219 & 0.66 & 0.701 & 0.895 \\
\hline S\&P BVL & Peru & 0.533 & 0.836 & 0.822 & 0.378 & 0.769 & 0.64 & 0.822 & 0.876 & 0.333 & 0.742 & 0.556 \\
\hline Colombia Colcap & Colombia & 0.507 & 0.047 & 0.082 & 0.32 & 0.125 & 0.868 & 0.023 & 0.59 & 0.213 & 0.34 & 0.068 \\
\hline $\mathrm{BCT}$ & Costa Rica & 0.637 & 0.223 & 0.792 & 0.404 & 0.792 & 0.792 & 0.056 & 0.334 & 0.323 & 0.598 & 0.302 \\
\hline Bermuda In. & Bermuda & 0.699 & 0.177 & 0.236 & 0.236 & 0.597 & 0.751 & 0.307 & 0.519 & 0.474 & 0.716 & 0.319 \\
\hline B. Eur. 500 & Europe & 0.409 & 0.337 & 0.839 & 0.797 & 0.039 & 0.133 & 0.044 & 0.441 & 0.19 & 0.172 & 0.133 \\
\hline MSCI Europe & Europe & 0.275 & 0.2 & 0.788 & 0.788 & 0.035 & 0.235 & 0.11 & 0.862 & 0.335 & 0.367 & 0.11 \\
\hline S\&P Europe 350 & Europe & 0.261 & 0.248 & 0.862 & 0.716 & 0.038 & 0.261 & 0.125 & 0.987 & 0.42 & 0.42 & 0.11 \\
\hline DAX & Germany & 0.687 & 0.132 & 0.865 & 0.23 & 0.264 & 0.104 & 0.234 & 0.127 & 0.431 & 0.113 & 0.344 \\
\hline CAC 40 & France & 0.297 & 0.371 & 0.652 & 0.404 & 0.093 & 0.52 & 0.099 & 0.61 & 0.404 & 0.186 & 0.036 \\
\hline Ibex 35 & Spain & 0.284 & 0.6 & 0.464 & 0.311 & 0.297 & 0.796 & 0.54 & 0.83 & 0.935 & 0.62 & 0.483 \\
\hline FTSE 100 & U.K. & 0.259 & 0.317 & 0.798 & 0.419 & 0.088 & 0.481 & 0.02 & 0.929 & 0.33 & 0.788 & 0.121 \\
\hline FTSE All & U.K. & 0.816 & 0.171 & 0.051 & 0.788 & 0.031 & 0.092 & 0.004 & 0.03 & 0.109 & 0.079 & 0.001 \\
\hline Swiss Mar. In. & Switzerland & 0.128 & 0.988 & 0.219 & 0.131 & 0.744 & 0.816 & 0.641 & 0.465 & 0.913 & 0.988 & 0.988 \\
\hline FTSE MIB & Italy & 0.704 & 0.255 & 0.365 & 0.815 & 0.007 & 0.08 & 0.051 & 0.255 & 0.267 & 0.122 & 0.038 \\
\hline FTSE Italia All & Italy & 0.63 & 0.124 & 0.346 & 0.323 & 0.07 & 0.535 & 0.077 & 0.241 & 0.37 & 0.135 & 0.057 \\
\hline PSI All Share & Portugal & 0.191 & 0.697 & 0.792 & 0.586 & 0.465 & 0.732 & 0.153 & 0.25 & 0.197 & 0.244 & 0.375 \\
\hline Irish Overall & Ireland & 0.336 & 0.681 & 0.83 & 0.481 & 0.736 & 0.473 & 0.052 & 0.094 & 0.227 & 0.394 & 0.361 \\
\hline Iceland St. Exc. & Iceland & 0.813 & 0.19 & 0.024 & 0.829 & 0.307 & 0.392 & 0.115 & 0.051 & 0.085 & 0.198 & 0.212 \\
\hline Amsterdam In. & Netherlands & 0.946 & 0.171 & 0.646 & 0.528 & 0.252 & 0.227 & 0.003 & 0.387 & 0.488 & 0.094 & 0.164 \\
\hline Belgium 20 & Belgium & 0.615 & 0.084 & 0.905 & 0.791 & 0.203 & 0.405 & 0.01 & 0.276 & 0.284 & 0.667 & 0.098 \\
\hline Brussels St. Exc. & Belgium & 0.052 & 0.928 & 0.182 & 0.241 & 0.641 & 0.688 & 0.176 & 0.399 & 0.737 & 0.456 & 0.851 \\
\hline Luxemburg In. & Luxemburg & 0.912 & 0.537 & 0.496 & 0.58 & 0.384 & 0.304 & 0.056 & 0.764 & 0.125 & 0.937 & 0.987 \\
\hline OMX Cop. & Denmark & 0.063 & 0.633 & 0.327 & 0.2 & 0.352 & 0.651 & 0.782 & 0.314 & 0.98 & 0.421 & 0.669 \\
\hline
\end{tabular}




\begin{tabular}{|c|c|c|c|c|c|c|c|c|c|c|c|c|}
\hline OMX Helsinki & Finland & 0.141 & 0.446 & 0.994 & 0.807 & 0.13 & 0.201 & 0.923 & 0.093 & 0.134 & 0.473 & 0.223 \\
\hline Oslo All & Norway & 0.314 & 0.96 & 0.209 & 0.227 & 0.615 & 0.706 & 0.669 & 0.92 & 0.9 & 1 & 0.529 \\
\hline OMX All & Sweden & 0.325 & 0.222 & 0.09 & 0.195 & 0.418 & 0.28 & 0.341 & 0.214 & 0.13 & 0.991 & 0.596 \\
\hline Vienna St. Ex. & Austria & 0.574 & 0.67 & 0.509 & 0.449 & 0.561 & 0.756 & 0.112 & 0.352 & 0.214 & 0.801 & 0.229 \\
\hline Athens St. Exc. & Greece & 0.186 & 0.501 & 0.728 & 0.923 & 0.59 & 0.412 & 0.853 & 0.492 & 0.631 & 0.53 & 0.492 \\
\hline Warsaw St. Exc. & Poland & 0.876 & 0.179 & 0.405 & 0.905 & 0.365 & 0.833 & 0.087 & 0.268 & 0.245 & 0.602 & 0.151 \\
\hline Prague St. Exc. & Czech Rep. & 0.244 & 0.022 & 0.18 & 0.629 & 0.392 & 0.758 & 0.03 & 0.153 & 0.227 & 0.203 & 0.195 \\
\hline MICEX & Russia & 0.502 & 0.907 & 0.62 & 0.93 & 0.243 & 0.267 & 0.35 & 0.08 & 0.22 & 0.243 & 0.231 \\
\hline Budapest In. & Hungary & 0.848 & 0.26 & 0.978 & 0.481 & 0.978 & 0.589 & 0.284 & 0.268 & 0.641 & 0.934 & 0.136 \\
\hline Ukraine PFTS & Ukraine & 0.62 & 0.838 & 0.293 & 0.307 & 0.815 & 0.704 & 0.17 & 0.414 & 0.108 & 0.884 & 0.661 \\
\hline Kazakhstan In. & Kazakhstan & 0.605 & 0.47 & 0.81 & 0.389 & 0.654 & 0.605 & 0.191 & 0.491 & 0.27 & 0.81 & 1 \\
\hline Slovak Share & Slovakia & 0.66 & 0.119 & 0.02 & 0.758 & 0.693 & 1 & 0.062 & 0.965 & 0.272 & 0.312 & 0.983 \\
\hline Zagreb St. Exc. & Croatia & 0.74 & 0.244 & 0.431 & 0.431 & 0.868 & 0.648 & 0.561 & 0.229 & 0.967 & 0.772 & 0.74 \\
\hline Ljubljana In. & Slovenia & 0.945 & 0.206 & 0.597 & 0.395 & 0.28 & 0.872 & 0.63 & 0.124 & 0.801 & 0.663 & 0.124 \\
\hline Rep. Srpska In. & Serbia & 0.473 & 0.918 & 0.027 & 0.065 & 0.442 & 0.918 & 0.356 & 0.682 & 0.2 & 0.124 & 0.282 \\
\hline OMX Tallinn & Estonia & 0.706 & 0.019 & 0.021 & 1 & 0.352 & 0.05 & 0.022 & 0.003 & 0.119 & 0.008 & 0.102 \\
\hline MBI 10 & Macedonia & 0.194 & 0.977 & 0.507 & 0.403 & 0.624 & 0.112 & 0.471 & 0.312 & 0.665 & 0.089 & 0.061 \\
\hline OMX Riga & Latvia & 0.068 & 0.021 & 0.302 & 0.318 & 1 & 0.408 & 0.535 & 0.085 & 0.705 & 0.757 & 0.039 \\
\hline OMX Vilnius & Lithuania & 0.973 & 0.203 & 0.605 & 0.318 & 0.558 & 0.945 & 0.973 & 0.036 & 0.945 & 0.286 & 0.654 \\
\hline Bulgaria Ind & Bulgaria & 0.611 & 0.235 & 0.777 & 0.559 & 0.692 & 0.51 & 0.51 & 0.418 & 0.985 & 0.44 & 0.836 \\
\hline Borsa. Ist. 100 & Turkey & 0.058 & 0.06 & 0.756 & 0.096 & 0.043 & 0.256 & 0.009 & 0.01 & 0.244 & 0.166 & 0.017 \\
\hline Cyprus General & Cyprus & 0.624 & 0.931 & 0.751 & 0.665 & 0.312 & 0.1 & 0.708 & 0.583 & 0.544 & 0.795 & 0.624 \\
\hline Malta St. Exc. & Malta & 0.2 & 0.119 & 0.841 & 0.481 & 0.102 & 0.268 & 0.014 & 0.066 & 0.58 & 0.563 & 0.669 \\
\hline FTSE JSE All & S. Africa & 0.33 & 0.597 & 0.446 & 0.991 & 0.218 & 0.824 & 0.307 & 0.55 & 0.699 & 0.716 & 0.275 \\
\hline EGX 30 & Egypt & 0.137 & 0.484 & 0.838 & 0.255 & 0.54 & 0.559 & 0.051 & 0.129 & 0.64 & 0.748 & 0.414 \\
\hline MADEX & Morocco & 0.561 & 0.481 & 0.062 & 1 & 0.384 & 0.384 & 0.199 & 0.038 & 0.025 & 0.804 & 0.068 \\
\hline Tunisia St. Exc. & Tunes & 0.912 & 0.716 & 0.289 & 0.788 & 0.693 & 0.319 & 0.58 & 0.189 & 0.669 & 0.537 & 0.275 \\
\hline FTSE JSE Nam. & Namibia & 1 & 0.124 & 0.798 & 0.608 & 0.112 & 0.538 & 0.505 & 0.72 & 0.2 & 0.151 & 0.282 \\
\hline Botswana Gaborone & Botswana & 0.818 & 0.695 & 0.31 & 0.655 & 0.218 & 0.441 & 0.076 & 0.86 & 0.968 & 0.543 & 0.598 \\
\hline Nigerian Ind & Nigeria & 0.953 & 0.953 & 0.579 & 0.599 & 0.54 & 0.431 & 0.54 & 0.414 & 0.448 & 0.414 & 0.559 \\
\hline Tanzania All & Tanzania & 0.021 & 0.91 & 0.97 & 0.031 & 0.308 & 0.91 & 0.273 & 0.791 & 0.017 & 0.385 & 0.734 \\
\hline Nairobi Sec. All & Kenya & 0.931 & 1 & 0.063 & 1 & 0.796 & 0.796 & 1 & 0.489 & 0.667 & 0.34 & 0.667 \\
\hline Ghana Com. & Ghana & 0.818 & 0.818 & 0.31 & 0.31 & 0.818 & 0.31 & 0.818 & 0.485 & 0.24 & 0.937 & 0.394 \\
\hline Kuwait St. Exc. & Kuwait & 0.791 & 0.308 & 0.678 & 0.162 & 0.91 & 0.623 & 0.791 & 0.427 & 0.273 & 0.623 & 0.045 \\
\hline Tel Aviv St. Exc. & Israel & 0.015 & 0.712 & 0.112 & 0.816 & 0.816 & 0.712 & 0.67 & 0.222 & 0.522 & 0.861 & 0.574 \\
\hline Blom Ind & Lebanon & 0.407 & 0.087 & 0.024 & 0.529 & 0.314 & 0.466 & 0.9 & 0.138 & 0.092 & 0.651 & 0.209 \\
\hline Bahrain All & Bahrain & 0.918 & 0.918 & 0.918 & 0.608 & 0.918 & 0.608 & 0.505 & 0.356 & 0.305 & 1 & 0.72 \\
\hline Tadawull All & S. Arabia & 0.645 & 0.282 & 0.13 & 0.775 & 0.93 & 1 & 0.16 & 0.826 & 0.312 & 0.282 & 0.087 \\
\hline Amman St. Exc. & Jordan & 0.705 & 0.371 & 0.158 & 0.513 & 0.945 & 0.973 & 0.836 & 0.256 & 0.203 & 0.783 & 0.513 \\
\hline Muscat MSM 30 & Oman & 0.877 & 0.861 & 0.548 & 0.771 & 0.362 & 0.277 & 0.985 & 0.561 & 0.727 & 0.438 & 0.426 \\
\hline Blg. GCC 200 & GCCC & 0.948 & 0.264 & 0.793 & 0.555 & 0.896 & 0.646 & 0.511 & 0.694 & 0.264 & 0.646 & 0.115 \\
\hline QE All Share & Qatar & 0.473 & 0.427 & 0.85 & 0.678 & 0.91 & 0.308 & 0.241 & 0.623 & 0.308 & 0.385 & 0.212 \\
\hline Dubai F. M. G. & UAE & 0.798 & 0.2 & 0.412 & 0.238 & 0.682 & 0.608 & 0.838 & 0.72 & 0.124 & 0.878 & 0.101 \\
\hline Abu Dhabi G. & UAE & 0.59 & 0.229 & 0.804 & 0.431 & 0.709 & 0.481 & 0.431 & 0.934 & 0.068 & 0.74 & 0.281 \\
\hline M. SEMDEX & Mauritius & 0.096 & 0.762 & 0.641 & 0.193 & 0.967 & 0.915 & 0.103 & 0.031 & 0.863 & 0.98 & 0.915 \\
\hline Tokyo St. Ind. & Japan & 0.346 & 0.723 & 0.874 & 0.268 & 0.388 & 0.691 & 0.12 & 0.043 & 0.713 & 0.493 & 0.234 \\
\hline
\end{tabular}




\begin{tabular}{lllllllllllll} 
Nikkei 225 & Japan & 0.922 & 0.645 & 0.297 & 0.209 & 0.645 & 0.293 & 0.176 & 0.345 & 0.785 & 0.235 & 0.26 \\
NSE Nifty 50 & India & 0.756 & 0.592 & 0.604 & 0.299 & 0.426 & 0.836 & 0.201 & 0.945 & 0.511 & 0.917 & 0.863 \\
S\&P Sensex & India & 0.51 & 0.415 & 0.971 & 0.358 & 0.374 & 0.728 & 0.12 & 0.938 & 0.47 & 0.744 & 0.84 \\
HIS Index & HK - China & 0.463 & 0.454 & 0.076 & 0.307 & 0.223 & 0.356 & 0.607 & 0.722 & 0.892 & 0.064 & 0.454 \\
CSI 300 & M. China & 0.199 & 0.678 & 0.431 & 0.901 & 0.868 & 0.709 & 0.534 & 0.836 & 0.184 & 1 & 0.709 \\
Shanghai Comp. & M. China & 0.375 & 0.615 & 0.708 & 0.876 & 0.934 & 0.301 & 0.978 & 0.949 & 0.268 & 0.481 & 0.355 \\
Shenzhen Comp. & M. China & 0.194 & 0.509 & 0.655 & 0.522 & 0.509 & 0.985 & 0.244 & 0.742 & 0.146 & 0.742 & 0.954 \\
Kospi & South Korea & 0.627 & 0.239 & 0.17 & 0.604 & 0.779 & 0.331 & 0.364 & 0.905 & 0.837 & 0.285 & 0.517 \\
Bangkok SET & Thailand & 0.446 & 0.674 & 0.947 & 0.888 & 0.819 & 1 & 0.277 & 0.277 & 0.52 & 0.888 & 0.404 \\
Straits Time & Singapore & 0.318 & 0.039 & 0.558 & 0.783 & 0.113 & 0.513 & 0.085 & 0.428 & 0.449 & 0.046 & 0.068 \\
FTSE KLCI & Malaysia & 0.567 & 0.718 & 0.093 & 0.156 & 0.51 & 0.15 & 0.081 & 0.683 & 0.634 & 0.358 & 0.607 \\
Jakarta Ind. & Indonesia & 0.568 & 0.436 & 0.042 & 0.115 & 0.415 & 0.272 & 0.272 & 0.946 & 0.84 & 0.995 & 0.736 \\
Philippine Ind & Philippines & 0.6 & 0.674 & 0.004 & 0.158 & 0.923 & 0.234 & 0.284 & 0.501 & 0.429 & 0.631 & 0.959 \\
Karachi KSE100 & Pakistan & 0.6 & 0.674 & 0.004 & 0.158 & 0.923 & 0.234 & 0.284 & 0.501 & 0.429 & 0.631 & 0.959 \\
Sri Lanka Ind. & Sri Lanka & 0.692 & 0.311 & 0.814 & 0.317 & 0.888 & 0.103 & 0.372 & 0.825 & 0.634 & 0.424 & 0.087 \\
MSE top 20 & Mongolia & 0.022 & 0.42 & 0.962 & 0.912 & 0.812 & 0.764 & 0.693 & 0.693 & 0.812 & 0.764 & 0.261 \\
Laos Index & Laos & 0.589 & 1 & 0.009 & 0.132 & 0.093 & 0.31 & 0.31 & 0.041 & 0.485 & 0.818 & 0.041 \\
Ho Chi Minh In. & Vietnam & 0.491 & 0.371 & 0.513 & 0.215 & 0.449 & 0.605 & 0.428 & 0.335 & 0.679 & 0.535 & 0.918 \\
Australian All & Australia & 0.687 & 0.037 & 0.063 & 0.758 & 0.165 & 0.544 & 0.71 & 0.129 & 0.507 & 0.888 & 0.238 \\
S\&P/NZX All & New Zealand & 0.063 & 0.362 & 0.461 & 0.023 & 0.6 & 0.383 & 0.277 & 0.614 & 0.535 & 0.342 & 0.497 \\
\hline
\end{tabular}

Data source: Bloomberg

Table 3. Wilcoxon test comparing January returns with the rest of month (p-values) for the last 15 years

\begin{tabular}{|c|c|c|c|c|c|c|c|c|c|c|c|c|}
\hline Index & Jurisdiction & Feb & Mar & Apr & May & Jun & Jul & Aug & Sept & Oct & Nov & Dec \\
\hline S\&P 500 Index & U.S. & 0.089 & 0.868 & 0.481 & 0.534 & 0.320 & 0.678 & 0.804 & 0.362 & 0.901 & 0.901 & 0.836 \\
\hline Dow Jones Industrial & U.S. & 0.384 & 0.431 & 0.709 & 0.868 & 0.320 & 0.320 & 0.507 & 0.340 & 0.507 & 0.481 & 0.125 \\
\hline Nasdaq Composite & U.S. & 0.097 & 1.000 & 0.263 & 0.455 & 0.263 & 0.804 & 0.246 & 0.263 & 0.590 & 0.619 & 0.648 \\
\hline Nasdaq 100 & U.S. & 0.031 & 0.619 & 0.300 & 0.481 & 0.281 & 0.709 & 0.125 & 0.125 & 0.481 & 0.455 & 0.619 \\
\hline N.Y. Stock Ex Comp. & U.S. & 0.678 & 0.561 & 0.836 & 0.934 & 0.229 & 0.481 & 0.281 & 0.678 & 0.709 & 0.590 & 0.147 \\
\hline S\&P 100 & U.S. & 0.213 & 0.300 & 0.507 & 0.740 & 0.281 & 0.561 & 0.934 & 0.320 & 0.804 & 0.678 & 0.340 \\
\hline S\&P 1500 & U.S. & 0.106 & 0.709 & 0.431 & 0.561 & 0.340 & 0.648 & 0.804 & 0.431 & 0.967 & 0.934 & 0.868 \\
\hline Russell 1000 & U.S. & 0.106 & 0.772 & 0.455 & 0.590 & 0.340 & 0.678 & 0.934 & 0.431 & 0.967 & 0.868 & 0.901 \\
\hline Russell 2000 & U.S. & 0.590 & 0.836 & 0.619 & 0.590 & 0.590 & 0.431 & 0.868 & 0.281 & 0.836 & 0.534 & 0.868 \\
\hline Russell 3000 & U.S. & 0.097 & 0.678 & 0.455 & 0.590 & 0.340 & 0.678 & 0.868 & 0.407 & 1.000 & 0.967 & 0.901 \\
\hline S\&P Toronto Com. & Canada & 0.281 & 0.740 & 0.967 & 0.455 & 0.836 & 0.868 & 0.709 & 0.709 & 0.507 & 0.836 & 0.868 \\
\hline S\&P BMV Mexico & Mexico & 0.868 & 0.047 & 0.678 & 0.507 & 0.431 & 0.455 & 0.590 & 0.431 & 0.089 & 0.213 & 0.106 \\
\hline Bolsa de Valores Pan. & Panama & 0.362 & 0.772 & 0.431 & 0.590 & 0.967 & 0.115 & 0.125 & 0.320 & 0.362 & 0.016 & 0.062 \\
\hline Buenos Aires Stock Ex. & Argentina & 0.804 & 0.384 & 0.836 & 0.159 & 0.147 & 0.619 & 0.407 & 0.184 & 0.507 & 0.320 & 0.384 \\
\hline Ibovespa & Brazil & 0.590 & 0.135 & 0.171 & 0.481 & 0.062 & 0.199 & 0.125 & 0.934 & 0.115 & 0.106 & 0.159 \\
\hline Caracas Stock Exc. & Venezuela & 0.320 & 0.281 & 0.590 & 0.481 & 0.340 & 0.300 & 0.184 & 0.804 & 0.263 & 0.648 & 0.619 \\
\hline S\&P BVL Peru & Peru & 0.934 & 0.534 & 0.619 & 0.534 & 0.507 & 0.619 & 0.648 & 0.455 & 0.340 & 0.507 & 0.431 \\
\hline Colombia Colcap & Colombia & 0.507 & 0.047 & 0.082 & 0.320 & 0.125 & 0.868 & 0.023 & 0.590 & 0.213 & 0.340 & 0.068 \\
\hline BCT Costa Rica & Costa Rica & 0.663 & 0.787 & 0.362 & 0.772 & 0.678 & 0.246 & 0.431 & 0.804 & 0.934 & 0.619 & 0.934 \\
\hline Bermuda Stock Ex. & Bermuda & 0.804 & 0.047 & 0.125 & 0.125 & 0.934 & 0.619 & 0.340 & 0.362 & 0.263 & 0.740 & 0.362 \\
\hline Bloomberg Europe 500 & Europe & 0.281 & 0.199 & 0.740 & 1.000 & 0.281 & 0.740 & 0.340 & 0.772 & 0.384 & 0.868 & 0.407 \\
\hline MSCI Europe Index & Europe & 0.362 & 0.171 & 0.709 & 1.000 & 0.263 & 0.590 & 0.300 & 0.868 & 0.481 & 0.868 & 0.263 \\
\hline
\end{tabular}




\begin{tabular}{|c|c|c|c|c|c|c|c|c|c|c|c|c|}
\hline S\&P Europe 350 & Europe & 0.340 & 0.263 & 0.678 & 0.967 & 0.281 & 0.561 & 0.340 & 0.836 & 0.534 & 0.901 & 0.281 \\
\hline DeustcheBorse DAX & Germany & 0.340 & 0.407 & 0.229 & 0.740 & 0.300 & 0.407 & 0.481 & 0.619 & 0.868 & 0.934 & 0.507 \\
\hline CAC 40 & France & 0.362 & 0.135 & 0.836 & 0.901 & 0.125 & 0.648 & 0.407 & 0.804 & 0.648 & 0.619 & 0.159 \\
\hline Ibex 35 & Spain & 0.934 & 0.213 & 0.836 & 0.481 & 0.042 & 0.678 & 0.213 & 0.868 & 0.590 & 0.534 & 0.246 \\
\hline FTSE 100 & U.K. & 0.362 & 0.263 & 0.772 & 0.868 & 0.213 & 0.740 & 0.213 & 0.229 & 0.384 & 0.507 & 0.340 \\
\hline FTSE All Share & U.K. & 0.320 & 0.320 & 0.836 & 0.772 & 0.199 & 0.709 & 0.263 & 0.362 & 0.384 & 0.590 & 0.455 \\
\hline Swiss Market Index & Switzerland & 0.199 & 0.171 & 0.648 & 0.901 & 0.407 & 0.804 & 0.455 & 0.678 & 0.561 & 0.934 & 0.246 \\
\hline FTSE MIB Borsa & Italy & 0.481 & 0.159 & 0.534 & 0.561 & 0.047 & 0.481 & 0.171 & 0.534 & 0.507 & 0.320 & 0.068 \\
\hline PSI All Share & Portugal & 0.246 & 0.967 & 0.619 & 0.740 & 0.481 & 0.901 & 0.362 & 0.901 & 0.868 & 0.320 & 0.281 \\
\hline Irish Stock Ex. Overall & Ireland & 0.281 & 0.678 & 0.678 & 0.384 & 0.678 & 0.481 & 0.184 & 0.836 & 0.507 & 0.836 & 0.534 \\
\hline Iceland Stock Exc. & Iceland & 0.772 & 0.320 & 0.062 & 0.772 & 0.263 & 0.590 & 0.229 & 0.089 & 0.184 & 0.619 & 0.106 \\
\hline Amsterdam Stock Ex. & Netherlands & 0.648 & 0.125 & 0.836 & 1.000 & 0.362 & 0.967 & 0.229 & 1.000 & 0.967 & 0.772 & 0.836 \\
\hline Belgium 20 & Belgium & 0.407 & 0.115 & 0.320 & 0.561 & 0.229 & 0.836 & 0.068 & 0.648 & 0.229 & 0.281 & 0.229 \\
\hline Brussels St. Exc. & Belgium & 0.097 & 0.619 & 0.901 & 0.934 & 0.772 & 0.678 & 0.199 & 0.340 & 0.678 & 1.000 & 0.481 \\
\hline Luxemburg Sto. Exc. & Luxemburg & 0.901 & 0.481 & 0.561 & 0.934 & 0.507 & 0.590 & 0.171 & 0.678 & 0.147 & 0.740 & 0.934 \\
\hline OMX Copenhagen & Denmark & 0.171 & 0.561 & 0.561 & 0.340 & 0.362 & 0.678 & 0.836 & 0.362 & 0.709 & 0.384 & 0.868 \\
\hline OMX Helsinki & Finland & 0.213 & 0.804 & 0.678 & 0.709 & 0.263 & 0.407 & 1.000 & 0.507 & 0.407 & 1.000 & 0.740 \\
\hline Oslo All Share & Norway & 0.481 & 0.901 & 0.159 & 0.362 & 0.678 & 0.561 & 1.000 & 0.590 & 0.772 & 0.481 & 0.740 \\
\hline OMX Stockholm All & Sweden & 0.648 & 0.184 & 0.648 & 0.619 & 0.340 & 0.184 & 0.125 & 0.678 & 0.028 & 0.901 & 0.320 \\
\hline Vienna Stock Exchange & Austria & 0.590 & 0.407 & 0.868 & 0.967 & 0.213 & 0.772 & 0.199 & 0.320 & 0.481 & 0.648 & 0.229 \\
\hline Athens St. Exc. Gen. & Greece & 0.455 & 0.481 & 0.901 & 0.967 & 0.320 & 0.934 & 0.868 & 0.678 & 1.000 & 0.648 & 0.263 \\
\hline Warsaw St. Exc. & Poland & 0.836 & 0.106 & 0.534 & 0.934 & 0.125 & 0.804 & 0.507 & 0.740 & 0.431 & 0.125 & 0.213 \\
\hline Prague St. Exc. & Czech Rep. & 0.619 & 0.062 & 0.075 & 0.740 & 0.135 & 0.534 & 0.082 & 0.199 & 0.115 & 0.125 & 0.082 \\
\hline & & 0.263 & 0.455 & 0.246 & 0.455 & 0.300 & 0.229 & 0.246 & 0.125 & 0.171 & 0.507 & 0.199 \\
\hline Budapest St. Exc. & Hungary & 0.709 & 0.300 & 0.772 & 0.740 & 0.836 & 0.772 & 0.740 & 0.481 & 0.561 & 0.901 & 0.213 \\
\hline & & 0.804 & 0.534 & 0.619 & 0.868 & 0.648 & 0.709 & 0.159 & 0.384 & 0.038 & 0.868 & 0.619 \\
\hline Kazakhstan St. Exc. & Kazakhstan & 0.934 & 0.229 & 0.934 & 0.619 & 0.561 & 0.340 & 0.199 & 0.407 & 0.184 & 0.678 & 0.868 \\
\hline Slovak Share & Slovakia & 0.246 & 0.089 & 0.047 & 0.246 & 0.340 & 0.229 & 0.125 & 0.678 & 0.147 & 0.051 & 0.740 \\
\hline Zagreb St. Exc. & Croatia & 0.740 & 0.147 & 0.431 & 0.431 & 0.868 & 0.648 & 0.561 & 0.229 & 0.967 & 0.772 & 0.740 \\
\hline OMX Tallinn & Estonia & 0.362 & 0.034 & 0.082 & 0.407 & 0.300 & 0.147 & 0.115 & 0.020 & 0.281 & 0.047 & 0.135 \\
\hline OMX Riga & Latvia & 0.097 & 0.031 & 0.678 & 0.281 & 0.619 & 0.804 & 0.678 & 0.047 & 0.772 & 0.534 & 0.062 \\
\hline OMX Vilnius & Lithuania & 0.868 & 0.300 & 0.740 & 0.407 & 0.246 & 0.481 & 0.740 & 0.125 & 0.740 & 0.455 & 0.901 \\
\hline Bulgaria St. Exc. & Bulgaria & 0.678 & 0.263 & 0.407 & 0.384 & 1.000 & 0.455 & 0.481 & 0.300 & 0.836 & 0.534 & 0.934 \\
\hline The BorsaIstambul 100 & Turkey & 0.455 & 0.023 & 0.590 & 0.068 & 0.051 & 0.678 & 0.184 & 0.159 & 0.199 & 0.281 & 0.056 \\
\hline Malta St. Exc. & Malta & 0.868 & 0.038 & 0.648 & 0.384 & 0.213 & 0.507 & 0.042 & 0.023 & 0.967 & 0.934 & 0.836 \\
\hline FTSE JSE Africa All & S. Africa & 0.089 & 0.648 & 0.967 & 0.619 & 0.709 & 0.804 & 1.000 & 0.561 & 0.709 & 0.772 & 1.000 \\
\hline Egyptian Exc. EGX 30 & Egypt & 0.246 & 0.229 & 0.772 & 0.320 & 0.507 & 0.384 & 0.082 & 0.229 & 0.901 & 0.967 & 0.407 \\
\hline MADEX Casablanca & Morocco & 0.561 & 0.481 & 0.062 & 1.000 & 0.384 & 0.384 & 0.199 & 0.038 & 0.025 & 0.804 & 0.068 \\
\hline Tunisia St. Exc. & Tunes & 0.740 & 0.678 & 0.384 & 0.967 & 0.836 & 0.534 & 0.678 & 0.125 & 0.740 & 0.320 & 0.115 \\
\hline Botswana Gaborone & Botswana & 0.678 & 0.740 & 1.000 & 0.709 & 0.648 & 0.709 & 0.115 & 0.836 & 0.772 & 1.000 & 0.740 \\
\hline Nigerian Sto. Exc. & Nigeria & 0.031 & 0.023 & 0.003 & 0.025 & 0.042 & 0.003 & 0.340 & 0.320 & 0.147 & 0.018 & 0.534 \\
\hline Tel Aviv St. Exc. & Israel & 0.042 & 0.868 & 0.068 & 0.590 & 0.709 & 0.678 & 0.678 & 0.534 & 1.000 & 0.648 & 0.648 \\
\hline Blom Stock Index & Lebanon & 0.836 & 0.590 & 0.023 & 0.934 & 0.455 & 0.648 & 0.455 & 1.000 & 0.740 & 0.229 & 0.619 \\
\hline Tadawull All Share & Saudi Arabia & 0.619 & 0.340 & 0.590 & 0.868 & 1.000 & 0.709 & 0.213 & 0.561 & 0.089 & 0.171 & 0.106 \\
\hline Amman St. Exc. & Jordan & 0.678 & 0.619 & 0.246 & 0.590 & 0.934 & 0.967 & 0.590 & 0.184 & 0.362 & 0.967 & 1.000 \\
\hline Muscat MSM 30 & Oman & 0.804 & 0.507 & 0.481 & 0.648 & 0.431 & 0.125 & 0.836 & 1.000 & 0.740 & 0.159 & 0.184 \\
\hline
\end{tabular}




\begin{tabular}{|c|c|c|c|c|c|c|c|c|c|c|c|c|}
\hline Abu Dhabi General & UAE & 0.590 & 0.229 & 0.804 & 0.431 & 0.709 & 0.481 & 0.431 & 0.934 & 0.068 & 0.740 & 0.281 \\
\hline Mauritius SEMDEX & Mauritius & 0.281 & 0.868 & 0.709 & 0.082 & 0.934 & 0.934 & 0.340 & 0.097 & 0.709 & 0.772 & 0.804 \\
\hline Tokyo St. Exc. Ind. & Japan & 0.836 & 0.619 & 0.507 & 0.709 & 0.590 & 0.481 & 0.171 & 0.561 & 0.868 & 0.804 & 1.000 \\
\hline Nikkei 225 & Japan & 0.934 & 0.836 & 0.362 & 0.648 & 0.648 & 0.340 & 0.097 & 0.300 & 0.967 & 0.590 & 0.804 \\
\hline NSE Nifty 50 & India & 0.934 & 0.455 & 0.740 & 0.281 & 0.934 & 0.648 & 0.590 & 0.481 & 0.362 & 0.678 & 1.000 \\
\hline S\&P BSE Sensex & India & 0.770 & 0.540 & 0.815 & 0.559 & 0.770 & 0.579 & 0.280 & 0.953 & 1.000 & 0.661 & 0.930 \\
\hline HIS Index & HK - China & 0.772 & 0.018 & 0.836 & 0.678 & 0.171 & 0.678 & 0.901 & 1.000 & 0.534 & 0.590 & 0.097 \\
\hline CSI 300 & M. China & 0.199 & 0.678 & 0.431 & 0.901 & 0.868 & 0.709 & 0.534 & 0.836 & 0.184 & 1.000 & 0.709 \\
\hline Shanghai Comp. & M. China & 0.184 & 0.481 & 0.619 & 0.836 & 0.619 & 0.507 & 0.384 & 0.804 & 0.246 & 0.678 & 0.534 \\
\hline Shenzhen Comp. & M. China & 0.068 & 0.868 & 0.561 & 0.648 & 0.590 & 0.967 & 0.678 & 0.772 & 0.229 & 0.868 & 0.740 \\
\hline Korea St. Exc.Kospi & South Korea & 0.709 & 0.147 & 0.772 & 0.868 & 1.000 & 0.967 & 0.590 & 0.836 & 0.709 & 0.561 & 0.229 \\
\hline Bangkok SET & Thailand & 0.362 & 0.229 & 0.868 & 0.619 & 0.455 & 0.678 & 0.384 & 0.934 & 0.125 & 0.804 & 0.135 \\
\hline Straits Time & Singapore & 0.407 & 0.034 & 0.229 & 0.561 & 0.263 & 0.868 & 0.213 & 0.901 & 0.481 & 0.062 & 0.082 \\
\hline FTSE Bursa KLCI & Malaysia & 0.362 & 0.097 & 0.431 & 0.901 & 0.031 & 0.648 & 0.082 & 0.648 & 0.340 & 0.300 & 0.340 \\
\hline Jakarta St. Exc. Ind. & Indonesia & 0.901 & 0.281 & 0.147 & 0.481 & 0.740 & 0.934 & 0.075 & 0.967 & 0.619 & 0.320 & 0.199 \\
\hline Philippine St. Exc. & Philippines & 0.619 & 0.199 & 0.028 & 0.772 & 0.320 & 0.199 & 0.901 & 0.507 & 0.709 & 0.362 & 0.362 \\
\hline Karachi KSE100 & Pakistan & 0.431 & 0.229 & 0.804 & 0.431 & 0.263 & 0.384 & 0.135 & 0.147 & 0.619 & 0.159 & 0.068 \\
\hline Sri Lanka Ind. & Sri Lanka & 0.934 & 0.868 & 0.804 & 0.199 & 0.619 & 0.023 & 0.384 & 0.455 & 0.678 & 0.199 & 0.147 \\
\hline MSE top 20 & Mongolia & 0.082 & 0.340 & 0.772 & 0.709 & 0.967 & 0.772 & 0.590 & 0.407 & 0.590 & 0.507 & 0.340 \\
\hline Ho Chi Minh St. In. & Vietnam & 0.534 & 0.407 & 0.678 & 0.648 & 0.934 & 0.901 & 0.320 & 0.199 & 0.901 & 0.407 & 0.740 \\
\hline Australian All Or. In. & Australia & 0.901 & 0.125 & 0.051 & 0.507 & 0.507 & 0.455 & 0.561 & 0.097 & 0.772 & 0.171 & 0.740 \\
\hline S\&P/NZX All index & New Zealand & 0.147 & 0.229 & 0.135 & 0.362 & 0.340 & 1.000 & 1.000 & 0.246 & 0.934 & 0.619 & 0.340 \\
\hline
\end{tabular}

Data source: Bloomberg

Table 4. Kruskal-Wallis probability tables

\begin{tabular}{|c|c|c|c|c|c|c|c|}
\hline Name & Ticker & $\begin{array}{c}\text { Full } \\
\text { series }\end{array}$ & $\begin{array}{c}\text { Last } 15 \\
\text { years }\end{array}$ & Name & Ticker & $\begin{array}{c}\text { Full } \\
\text { series }\end{array}$ & $\begin{array}{c}\text { Last } 15 \\
\text { years }\end{array}$ \\
\hline S\&P 500 & SPX & 0.0835 & 0.4622 & Ljubljana Exc. Ind. & SBITOP & 0.2967 & $*$ \\
\hline Dow Jones Industrial & INDU & 0.0020 & 0.1999 & Rep. SrpskaInd. & BIRS & 0.1192 & * \\
\hline Nasdaq Composite & CCMP & 0.8294 & 0.5898 & OMX Tallinn Ind. & TALSE & 0.0070 & 0.4359 \\
\hline Nasdaq 100 & NDX & 0.7929 & 0.176 & MBI 10 Ind. & MBI & 0.1151 & * \\
\hline N.Y. Stock Ex Comp. & NYA & 0.2297 & 0.4391 & OMX Riga Ind. & RIGSE & 0.1218 & 0.0829 \\
\hline S\&P 100 & OEX & 0.2793 & 0.2742 & OMX Vilnius Ind. & VILSE & 0.4460 & 0.4415 \\
\hline S\&P 1500 & SPR & 0.2764 & 0.4772 & Bulgaria St. Exc. & SOFIX & 0.7399 & 0.6701 \\
\hline Russell 1000 & RIY & 0.4163 & 0.4464 & BorsaIstambul 100 & XU100 & 0.2077 & 0.2056 \\
\hline Russell 2000 & RTY & 0.3885 & 0.765 & Cyprus Gen Exc. & CYSMMAPA & 0.3863 & * \\
\hline Russell 3000 & RAY & 0.429 & 0.5147 & Malta St. Exc. Ind. & MALTEX & 0.2197 & 0.0400 \\
\hline S\&P Toronto Com. Ind. & SPTSX & 0.0037 & 0.6951 & FTSEJSE Africa All & JALSH & 0.5511 & 0.3757 \\
\hline S\&P BMV Mexico Ind. & MEXBOL & 0.3131 & 0.393 & Egyptian EGX 30 & EGX30 & 0.0607 & 0.0624 \\
\hline Bolsa de Valores Pan. & BVPSBVP & 0.3441 & 0.3528 & $\begin{array}{l}\text { MADEX } \\
\text { Casablanca }\end{array}$ & MOSEMDX & 0.1005 & 0.1005 \\
\hline Buenos Aires Ind. & MERVAL & 0.284 & 0.6235 & Tunisia St. Exc. Ind. & TUSISE & 0.5602 & 0.5449 \\
\hline Ibovespa Ind. & IBOV & 0.9127 & 0.4844 & FTSE JSE Namibia & FTN098 & 0.3473 & * \\
\hline Chile 65 Ind. & CHILE65 & 0.1564 & $*$ & Botswana Gaborone & BGSMDC & 0.1261 & 0.6382 \\
\hline Caracas St. Exc. Ind. & IBVC & 0.4893 & 0.9816 & Nigerian Sto. Exc. & NGSEINDX & 0.0269 & 0.0255 \\
\hline S\&P BVL Peru Ind. & SPBLPGPT & 0.9048 & 0.9929 & Tanzania All Share & DARSDSEI & 0.0275 & * \\
\hline Colombia Colcap Ind. & COLCAP & 0.3908 & 0.3908 & Nairobi Exc. All & NSEASI & 0.4341 & * \\
\hline BCT Costa Rica Ind. & CRSMBCT & 0.8017 & 0.9302 & Ghana Composite & GGSECI & 0.5366 & * \\
\hline Bermuda Stock Ex. & BSX & 0.2968 & 0.4588 & Kuwait St. Exc. In & SECTMIND & 0.4231 & * \\
\hline Bloomberg Europe 500 & BE500 & 0.0904 & 0.4593 & TelAviv St. Exc. & TA-125 & 0.1009 & 0.0918 \\
\hline
\end{tabular}




\begin{tabular}{|c|c|c|c|c|c|c|c|}
\hline MSCI Europe Ind. & MXEU & 0.0953 & 0.4528 & Blom Stock Index & BLOM & 0.1714 & 0.2673 \\
\hline S\&P Europe 350 Ind. & SPEURO & 0.0956 & 0.5236 & Bahrain Bourse All & BHSEASI & 0.7358 & * \\
\hline DeustcheBorse DAX & DAX & 0.0612 & 0.2732 & Tadawull All Share & SASEID & 0.7182 & 0.7326 \\
\hline CAC 40 Ind. & CAC & 0.0993 & 0.0001 & Amman St. Exc. & JOSMGNFF & 0.3991 & 0.5056 \\
\hline Ibex 35 Ind. & IBEX & 0.6770 & 0.4845 & Muscat MSM 30 & MSM30 & 0.5979 & 0.1575 \\
\hline FTSE 100 Ind. & UKX & 0.0358 & 0.0545 & Bberg GCC 200 & BGCC200 & 0.1357 & $*$ \\
\hline FTSE All Share Ind. & ASX & 0.0103 & 0.0903 & QE All Share Ind. & QEAS & 0.0915 & * \\
\hline Swiss Market Ind. & SMI & 0.4326 & 0.434 & Dubai Fin. Mar. Ind. & DFMGI & 0.1759 & $*$ \\
\hline FTSE MIB BorsaItaliana & FTSEMIB & 0.1169 & 0.4294 & Abu Dhabi General & ADSMI & 0.0674 & 0.0674 \\
\hline FTSE Italia All Share & ITLMS & 0.3817 & $*$ & Mauritius SEMDEX & SEMDEX & 0.1966 & 0.5281 \\
\hline PSI All Share Ind. & BVLX & 0.2533 & 0.6323 & Tokyo St. Exc. Ind. & TPX & 0.0618 & 0.6331 \\
\hline Irish Stock Ex. Overall & ISEQ & 0.0548 & 0.5013 & Nikkei 225 Ind. & NKY & 0.1395 & 0.4443 \\
\hline Iceland Stock Exc. Ind. & ICEXI & 0.1467 & 0.3665 & NSE Nifty 50 Ind. & NIFTY & 0.6363 & 0.6352 \\
\hline Amsterdam Stock Ex. & AEX & 0.0825 & 0.7233 & S\&P BSE Sensex & SENSEX & 0.3559 & 0.9332 \\
\hline Belgium 20 Ind. & BEL20 & 0.1872 & 0.4421 & HIS Index Ind. & HSI & 0.0069 & 0.0558 \\
\hline Brussels St. Exc. Belgian & BELSTK & 0.3395 & 0.5831 & CSI 300 Ind. & SHSZ300 & 0.5993 & 0.5993 \\
\hline Luxemburg Sto. Exc. & LUXXX & 0.3395 & 0.7235 & Shanghai Comp. & SHCOMP & 0.5402 & 0.5488 \\
\hline OMX Copenhagen & KAX & 0.3074 & 0.4715 & Shenzhen Comp. & SZCOMP & 0.3397 & 0.4412 \\
\hline OMX Helsinki Ind. & HEX & 0.1610 & 0.6601 & Kospi & KOSPI & 0.216 & 0.7547 \\
\hline Oslo All Share Ind. & OSEAX & 0.4304 & 0.6236 & Bangkok SET Ind. & SET & 0.9354 & 0.8611 \\
\hline OMX Stockholm All & SAX & 0.0147 & 0.2498 & Straits Time Ind. & STI & 0.0678 & 0.0202 \\
\hline Vienna Stock Exchange & WBI & 0.1059 & 0.5944 & FTSE Bursa KLCI & FBMKLCI & 0.0177 & 0.0579 \\
\hline Athens St. Exc. General & ASE & 0.4789 & 0.7365 & Jakarta Stock Exc. & $\mathrm{JCI}$ & 0.0778 & 0.0514 \\
\hline Warsaw St. Exc. Ind. & WIG & 0.6257 & 0.7541 & Philippine St. Exc. & PCOMP & 0.0443 & 0.1797 \\
\hline Prague St. Exc. Ind. & $\mathrm{PX}$ & 0.0112 & 0.2971 & Karachi KSE100 & KSE100 & 0.3742 & 0.7760 \\
\hline MICEX Ind. & INDEXCF & 0.7925 & 0.8423 & Sri Lanka Colombo & CSEALL & 0.1497 & 0.0019 \\
\hline Budapest St. Exc. Ind. & BUX & 0.3000 & 0.6054 & MSE top 20 Ind. & MSETOP & 0.0997 & 0.0987 \\
\hline Ukraine PFTS Ind. & PFTS & 0.1164 & 0.1496 & Laos Composite & LSXC & 0.0230 & $*$ \\
\hline & & & & Ind. & & & \\
\hline Kazakhstan St. Exc. & KZKAK & 0.6398 & 0.6789 & Ho Chi Minh St. & VNINDEX & 0.8557 & 0.7380 \\
\hline Slovak Share Ind. & SKSM & 0.1011 & 0.3488 & Australian All Ind. & AS30 & 0.0382 & 0.0760 \\
\hline Zagreb St. Exc. Ind. & CRO & 0.7734 & 0.6876 & S\&P/NZX All Ind. & NZSE & 0.0125 & 0.0334 \\
\hline
\end{tabular}

*No data available for the period requested

Data source: Bloomberg

\section{Results}

The average returns per month for all the indexes analyzed as well as their standard deviations can be found in Appendix 1. These results, for an easier visualization, are presented grouped according to geographical characteristics and divided into five regions: Americas, Western Europe, Eastern Europe, Middle East \& Africa and Asia \& Oceania.

\subsection{Wilcoxon (Entire Time Series)}

Using the entire time series available in Bloomberg for the previously mentioned 106 indexes and after making the number of data points for each month equal a total of 66 indexes, representing $62.3 \%$ of the total, were found to have no statically different returns in the month of January compared to all the other 11 months of the year, 27 indexes had one month with returns statistically different from January, 8 with 2 months, 2 with 3 months, 2 with 4 months and 1 with 6 months. In most of the cases then there was no statistically significant difference. These results can be found in Table 2. The index with 6 months of statistically different returns compared to the returns of January was the OMX Tallin, an index representing the Estonian stock market. The median return, point estimate, for the OMX Tallin index is negative in January and statistically significantly lower than in March, April, July, August, September and November. The point estimates for the median in all these months were positive. These results would suggest that the returns in January for the OMX Index have been lower than the results in several other months. The January Effect is typically understood as the opposite effect with January having bigger returns than other months. The two indexes with 4 months statistically different from the results in January were the FTSE All Share Index (U.K.) and 
the BorsaIstambul 100 Index (Turkey). In both cases there was no significant difference in returns when comparing January to the rest of months in the first quarter. In the case of the U.K. index the point estimate for the median value of the return for the month of January was negative but small. The index had positive returns in all the 4 months with statistically significantly different returns (June, August, September and December). In the case of the Borsa Istambul Index the point estimates of the median followed a similar pattern than in the FTSE All Share index, with the same distribution of months (including the negative value for January and the positive value for all the other months), but with larger differences in the point estimated for the returns. The two indexes with 3 months with statistically different returns when compared to January are the Tanzanian All Share Index (Tanzania) and the Laos Composite Index (Laos). There is more disparity of results with these two cases. On one hand there is the case of the index for Tanzania. The results for this index were similar to the previous cases with the point estimate for the median coming negative but small in January followed by a few months of positive results (February, May and October). On the other hand there is the case of Laos, with rather strong results in January and three months of statistically significantly returns (April, September and December). It should be noted that the data series for the index is Laos is relatively short with only 6 years of returns available in Bloomberg (always fulfilling the requirement of having the same number of data points per month).

The eight indexes that have two months with returns statistically different from January can be seen in table 5. For none of the eight indexes there was a statistically significant difference between January and February. For a majority of indexes, 5 out of 8, there was a statistically significant difference between January and March. The results for the tests for these eight indexes (over the entire data series) seem to support that the results were statistically similar for most of the month. For the other two months the results in January seem to be weaker than in those two months for all these eight indexes.

Table 5. Indexes with 2 months

\begin{tabular}{ll}
\hline Dow Jones Industrial (U.S.) & Prague St. Exc. Index(Czech Rep.) \\
\hline Colombia Colcap (Colombia) & OMX Riga (Latvia) \\
\hline Bloomberg Europe 500 (Europe) & MADEX Casablanca (Morocco) \\
\hline FTSE MIB Borsa(Italy) & Straits Time Index (Singapore) \\
\hline
\end{tabular}

Of the 106 indexes there were 27 that had one month with statistically different results when compared to January. The list of these indexes can be found in Table 6. Of the 27 cases the point estimate for the median was higher than the month with different returns, according to the Wilcoxon test, in 11 cases. Those 11 indexes are the S\&P Toronto Composite, Bolsa de Valores de Panama Index, MSCI Europe Index, Tel Aviv Stock Exchange Index, Blom Stock Exchange Index, Jakarta Stock Exchange Index, Philippines Stock Exchange Index, Karachi Stock Exchange Index, MSE Top 20 and the Australian All Index. Only in four of these cases (S\&P Toronto Composite, Bolsa de Valores de Panama Index, Tel Aviv Stock Exchange Index and MSE Top 20 Index) the results in February seem to be lower than the returns in January. Another four indexes had lower returns in April with the rest of months with lower returns distributed across the first half of the year. There were no months with significantly lower returns than January in the second half of the year i.e., after June.

Table 6. Indexes with one month statistically different from January

\begin{tabular}{ll}
\hline S\&P 500 & OMX Vilnius \\
\hline N.Y. Stock Ex Comp. & Malta St. Exc. \\
\hline S\&P 100 & Kuwait St. Exc. \\
\hline S\&P Toronto Com. & Tel Aviv St. Exc. \\
\hline Bolsa de Valores de Panama Index & Blom Stock Index \\
\hline MSCI Europe Index & Mauritius SEMDEX \\
\hline S\&P Europe 350 & Tokyo St. Exc. Ind. \\
\hline CAC 40 & Jakarta Stock Exchange \\
\hline FTSE 100 & Philippine St. Exc. \\
\hline Iceland Stock Exc. & Karachi KSE100 \\
\hline Amsterdam Stock Ex. & MSE top 20 \\
\hline
\end{tabular}




\begin{tabular}{ll}
\hline Belgium 20 & Australian All Or. In. \\
\hline Slovak Share & S\&PNZX All index \\
\hline St. Exc. Ind. Rep.Srpska & \\
\hline
\end{tabular}

\subsection{Wilcoxon (Last 15 Years)}

The first thing to notice is that, as previously mentioned, when performing the analysis on the data series including the last 15 years of returns there is a shorter number of indexes. This is due to the fact that not all the indexes have 15 years of returns available. For a list of the indexes excluded from this analysis please see Table 1. Of the 90 indexes examined 67 , representing $74.4 \%$ of the total, presented no statistically significant different returns when using the Wilcoxon test. This represents a higher proportion that when analyzing the entire dataset available i.e., all the years, but excluding the indexes with less than 15 years of track record (the indexes in those two approaches would be the same but the time length of the data series would be different). When using the entire data series for the 90 indexes there is a total of 54 cases, representing approximately $60 \%$ of the cases, in which there is no statistically significant difference between the performance in January and the performance in any of the other months of the year. This seems to support the idea that the January-Effect might be dissipating over time.

When analyzing the last 15 years of data 17 indexes had returns statistically different in the month of January compared to another month in the same year. Three indexes had two months statistically different, two indexes three months and one index 7 months. The index that had 7 months with returns different from the returns on January was the Nigeria Stock Exchange Index. It would appear that in the case of Nigeria, during the last 15 years, that there are indications of a sizeable January Effect. The point estimate of the returns of this index is positive and statistically different from those on February, March, April, May, June, July and November with the majority of the point estimate for the returns in those months actually being negative.

The indexes that had three months of statisticallydifferent results were the OMX Tallim (Estonia) and the Malta Stock Exchange Index (Malta). Both of these indexes had negative median returns in the month of January. Similarly to the case when the entire data set is analyzed the Estonian index is one of the indexes that has the largest amount of months with statistically different returns when compared to the month of January. The returns in Estonian index seem to be negative for the month of January. It should be noted that the results in both cases, with the entire data set or only the last 15 years, the median of the returns is negative but the magnitude of the point estimate appears to decrease. This perhaps points to an inverse, but decreasing, January-Effect in the Estonian case. In the case of Malta the point estimate of the returns in January is also negative, with the months of March, August and September having statistically different results and positive point estimates for the median returns.

The indexes that have two months of statistically different results, compared to January, are the OMX Riga (Latvia), the Madex Casablanca (Morocco) and the Colcap Index (Colombia). In all these cases the point estimate of the median return for the month of January was negative or very close to zero while the point estimates for the months with statistically different returns were all positive. These results seem to indicate that there is no January-Effect in the traditional sense.

The 17 indexes that had one month of statistically different returns can be seen in table 7 . Of all these 17 indexes the returns were higher in January, compared to the other statistical different month, for five indexes. These indexes are the Nasdaq 100 Index (U.S.), the Tel Aviv Stock Exchange Index (Israel) and the Blom Index (Lebanon), Philippines Stock Exchange Index (Philippines) and Sri Lanka Colombo Index (Sri Lanka). In most of these cases the months of lower returns followed closely after January. In the cases of theNasdaq 100 Index and the Tal Aviv Stock Exchange Index it occurred in February and in the cases of the Blom Index and the Philippines Stock Exchange Index in April. The exception of this trend was the Sri Lanka Colombo Index with the significantly different month happening in July. The point estimates of the median returns of all these three month were negative. There are no indications of higher returns in January for the other 14 indexes. Most of the statistically different performances happened in the first half of the year with only four cases happening after June. The four cases with different performances after June were the Bolsa de Valores de Panama Index (November), the OMX Stockholm All Index (October), Ukraine PFTS Index (October) and the Sri Lanka Colombo Index (July).

Table 7. Indexes with one month statistically different from January (time series of 15 years)

\begin{tabular}{ll}
\hline Nasdaq 100 (U.S.) & Bora Istambul 100 (Turkey) \\
\hline S\&P BMV (Mexico) & Tel Aviv Stock Exchange Index (Israel) \\
\hline
\end{tabular}




\begin{tabular}{ll}
\hline Bolsa de Valores Index (Panama) & Blom Stock Index (Lebanon) \\
\hline Bermuda Stock Exchange Index (Bermuda) & HSI Index (Hong Kong - China) \\
\hline Ibex 35 (Spain) & Straits Time Index (Singapore) \\
\hline FTSE MIB Borsa (Italy) & FTSE Bursa KLCI (Malaysia) \\
\hline OMX Stockholm All (Sweden) & Philippine Stoc. Exc. Ind. (Philippines) \\
\hline Ukraine PFTS (Ukraine) & Sri Lanka Colombo Index (Sri Lanka) \\
\hline Slovak Share Index (Slovakia) & \\
\hline
\end{tabular}

\subsection{Kruskal-Wallis}

The results for the Kruskal-Wallis test, for both the entire time series as well as the last 15 years, can be found in Table 1. This test tries to determine if the returns for all the 12 months come from the same distribution. Therefore one $\mathrm{p}$ value is obtained for all the 12 months rather than having $11 \mathrm{p}$ values for the comparison of the months from February to December (comparing them to the returns in January).

At a 5\% confidence level an according to the results from the Kruskal-Wallis test $85.8 \%$ of the indexes , using the entire data set available, the returns for all the 12 months came from the same distribution. When the entire length of the time series is used but those indexes with less than 15 years of data are excluded then in $85.6 \%$ of the cases the data come from the same distribution. When only the last 15 years of data are used, and indexes with less than those 15 years of returns are included in the analysis, then $93.3 \%$ of the cases appear to come from the same distribution supporting the idea that the January-Effect is apparently dissipating over time. It should be noted that the test does not specifically compared the performance in January with the performance in the rest of months. The test analyzes the returns in all those months in its entirety. Using the entire time series the 15 indexes that do not appear to have the returns for all the months coming from the same distribution can be seen in Table 8. The 13 and 6 indexes for the entire time series length (excluding those indexes with less than 15 years of returns) as the case only including the last 15 years of returns respectively can be seen in Tables 9 and 10 .

Table 8. Entire time series, indexes with returns not coming from the same distribution

\begin{tabular}{ll}
\hline Dow Jones Industrial Ind. & Tanzania All Share Ind. \\
\hline S\&P Toronto Com. Ind. & HSI Index Ind. \\
\hline FTSE 100 Ind. & FTSE Bursa KLCI Ind. \\
\hline FTSE All Share Ind & Philippine St. Exc. Ind. \\
\hline OMX Stockholm All Ind. & Laos Securities Composite Ind. \\
\hline Prague St. Exc. Ind. & Australian All Ordinaries Ind. \\
\hline OMX Tallinn Ind. & S\&P/NZX All Ind. \\
\hline Nigerian Sto. Exc. Ind. & \\
\hline
\end{tabular}

Table 9. Entire time series (excluding indexes with less than 15 years of returns), indexes with returns not coming from the same distribution

\begin{tabular}{ll}
\hline Dow Jones Industrial Ind. & Nigerian Sto. Exc. Ind. \\
\hline S\&P Toronto Com. Ind. & HSI Index Ind. \\
\hline FTSE 100 Ind. & FTSE Bursa KLCI Ind. \\
\hline FTSE All Share Ind. & Philippine St. Exc. Ind. \\
\hline OMX Stockholm All Ind. & Australian All Ordinaries Ind. \\
\hline Prague St. Exc. Ind. & S\&P/NZX All Ind. \\
\hline OMX Tallinn Ind. & \\
\hline 10. 15 last years, indexes with returns not coming from the same distribution \\
\hline CAC 40 Ind. & Straits Time Ind. \\
\hline Malta St. Exc. Ind. & Sri Lanka Colombo Ind. \\
\hline Nigerian Sto. Exc. Ind. & S\&P/NZX All Ind. \\
\hline
\end{tabular}

\section{Conclusions}


While there are markets, such as for instance Nigeria, in which there continues to appear to exist the traditional January Effect there seems to be a global trend for the January Effect to gradually dissipate. This was been tested across the vast majority of countries with functioning equity markets. This conclusion is supported for both the results from the Wilcoxon and the Kruskal Wallis test. It was also observed that it is equally, if not more, prevalent an Inverse January Effect, with returns lower in January than in other months, than the traditional January Effect. The Estonian case is a good example of this trend of having lower returns in January than in several other months during the year. When there are statistically significant differences between the returns in January and of the months they tend to happen for month in the first half of the year. It should be noted that there are exceptions to this general trend. The assumption that the monthly return does not, in general, follow a normal distribution was tested with an Anderson Darling and a Lillie tests. In both cases the results point toward not accepting that assumption, which is in agreement with the existing literature. This non normality of returns is the reason why non parametric test were used to compare performance.

\section{References}

Anderson Lissa, Gerlach Jeffrey, \& DiTraglia Francis. (2008). Yes, Wall Street, there is a January effect! Evidence from laboratory auctions. Journal of Behavioral Finance, 8(1). http://dx.doi.org/10.1080/15427560709337012

Asteriou Dimitrios, \& Kavetsos Georgios. (2006). Testing for the existence of the January Effect in transition economies. Applied Financial Economics Letters, 2(6). http://dx.doi.org/10.1080/17446540600706817

Choudry Taufiq. (2011). Month of the year and January effect in pre-WWI stock returns: evidence from a non-linear GARCH model. International Journal of Finance and Economics, 6(1). http://dx.doi.org/10.1002/ijfe.142

Ciccone Stephen. (2011). Investor optimism, false hopes and the January effect. Journal of Behavioral Finance, 12(3). http://dx.doi.org/10.1080/15427560.2011.602197

Cooper Michael, McConnel John, \& Ovtchinnikov Alexei. (2006). The other January effect. Journal of Financial Economics, 82(2). http://dx.doi.org/10.1016/j.fincco.2006.03.001

DimaWaleed Hanna Alrabadi, \& Kamal Ahmad Al Qudah. (2012). Calendar anomalies: The case of Amman Stock Exchange. International Journal of Business and Management, 7(24).

Gamble Ralph (1993). The January effect and intergenerational transfers. The Quarterly Review of Economics and Finance, 33(3).

Gharaibeh Omar. (2017). The January effect from four Arabic market indices. International Journal of Academic Research in Accounting, Finance and Management Sciences, 7(1).

Gu Anthony Yanxiang. (2003). The declining January effect: evidence from the US equity market. The Quarterly Review of Economics and Finance, 43. https://doi.org/10.1016/S1062-9769(02)00160-6

GulerSevinc. (2013). January effect in stock returns: evidence from emerging markets. Interdisciplinary Journal of Contemporary Research in Business, 5(4).

Hashmi Arsalan Muhammad. (2014). January effect in Pakistan: A time series analysis. Journal of Management, Business and Economics, 9(1).

Haug Mark, \& Hirschey Mark. (2006). The January effect. Financial analysis Journal, 62(5). https://doi.org/10.2469/faj.v62.n5.4284

Haugen R.A, Lakonishok. (1988). The incredible January effect: the stock market's unsolved mystery. Dow Jones Irwin.

Haugen Robert, \& Torion Philippe. (1996). The January effect: still there after all these years. Financial Analyst Journal, 52(1).

He Ling, \& He Shao. (2011). Has the November effect replaced the January Effect in stock markets? Managerial and Decision Economics, 32.

Honghui Chen, \& Signal Vijay. (2004). All things considered, taxes drive the January effect. The Journal of Financial Research, XXVII(3).

Jones Charles, \& Jack Wilson. (1989). An analysis of the January effect in stocks. The Journal of Financial Research, XII(4). http://dx.doi.org/10.1111/j.1475-6803.1989.tb00527.x

KaurGurneet. (2017). The January Effect in the Indian Stock Market. International Journal of Recent Scienctific Research, 8(5). 
KlockShela, \& Bacon Frank. (2014). The January effect: a test of market efficiency. Journal of Business and Behavior Sciences, 26(3).

Li, Bin, \& Liu Benjamin. (2010). Monthly seasonality in the New Zealand Stock market. International Journal of Business Management and Economic Research, 1(1).

Li, Jingya, \& Gong, Jian. (2015). Volatility risk and January effect: evidence from Japan. International Journal of Economics and Finance, 7(6).

Mehdian Seyed, \& Perry Mark. (2012). Anomalies in US equity markets: a re-examination of the January effect. Applied Financial Economics, 12(2). http://dx.doi.org/10.1080/09603100110088067

Mehta Kiran, \& Chander Ramesh. (2009). Seasonality in Indian stock market: a re-examination of January effect. Asia-Pacific Journal of Management Research and Innovation, 5(4). https://doi.org/10.1177/097324700900500403

Moller Nicholas, \& Zilca Shlomo. (2008). The evolution of the January effect. Journal of Banking and Finance, 32(3). https://doi.org/10.1016/j.jbankfin.2007.06.009

PandeuSitaram, \& Samanta Amitava. (2016). An empirical analysis of January effect - evidence from Indian market. International Journal of Innovative Research Development, 5(7).

Patel Jayen. (2016). The January effect anomaly reexamined in stock returns. The Journal of Applied Business Research, 32(1).

Schultz Paul. (1985). Personal income taxes and the January Effect: small firm stock returns before the War Revenue Act of 1917: A note. The Journal of Finance, 40(1). https://doi.org/10.1111/j.1540-6261.1985.tb04954.x

SimbolonIka Pratiwi. (2015). January effect on stock returns in Indonesia: The unconditional method and the conditional method. International Business Management, 9(6).

Thaler Richard. (1987). The January effect. Economic Perspectives, 1(1).

Watchel, S. (1942). Certain observations on seasonal movements on stock prices. The Journal of Business, 15(2). 


\section{Appendix 1. Median returns and standard deviation by geography}

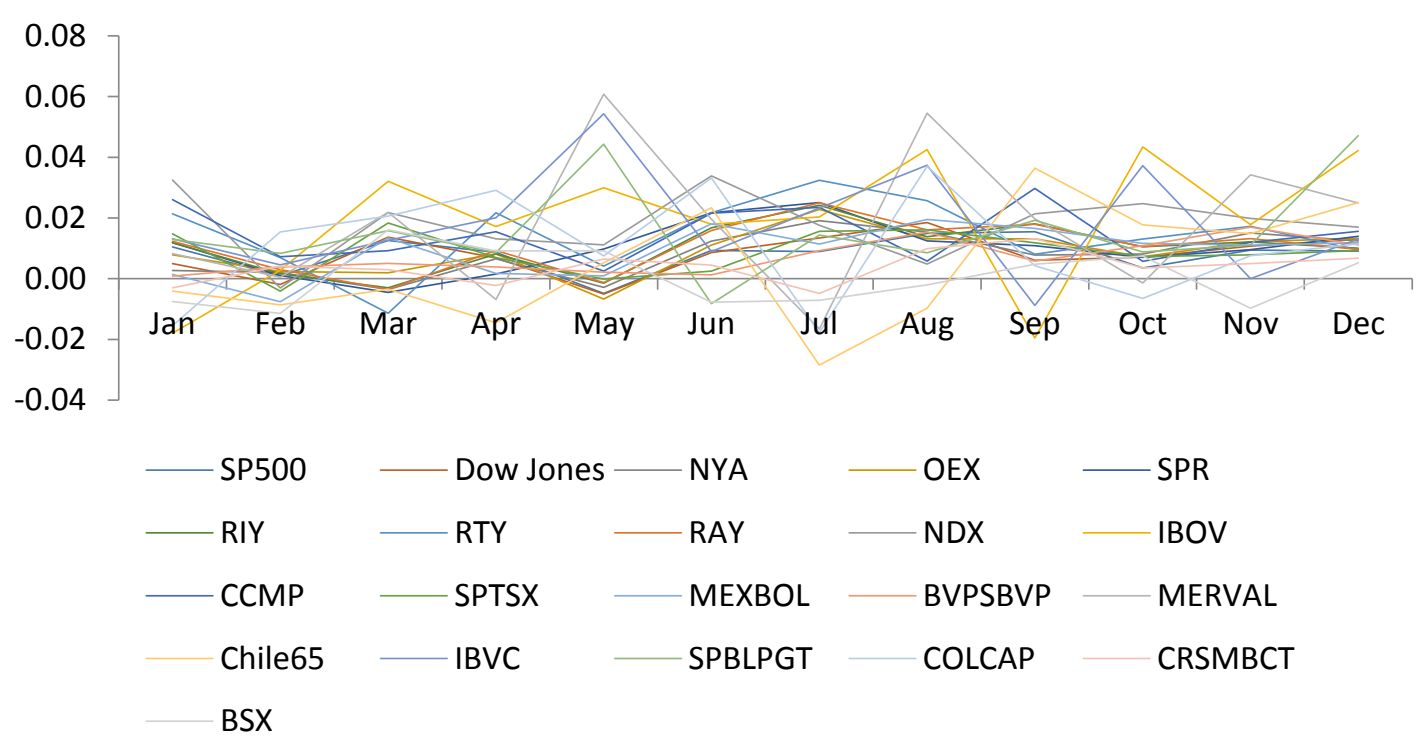

Figure 1. Average return per month - Americas

Data source: Bloomberg

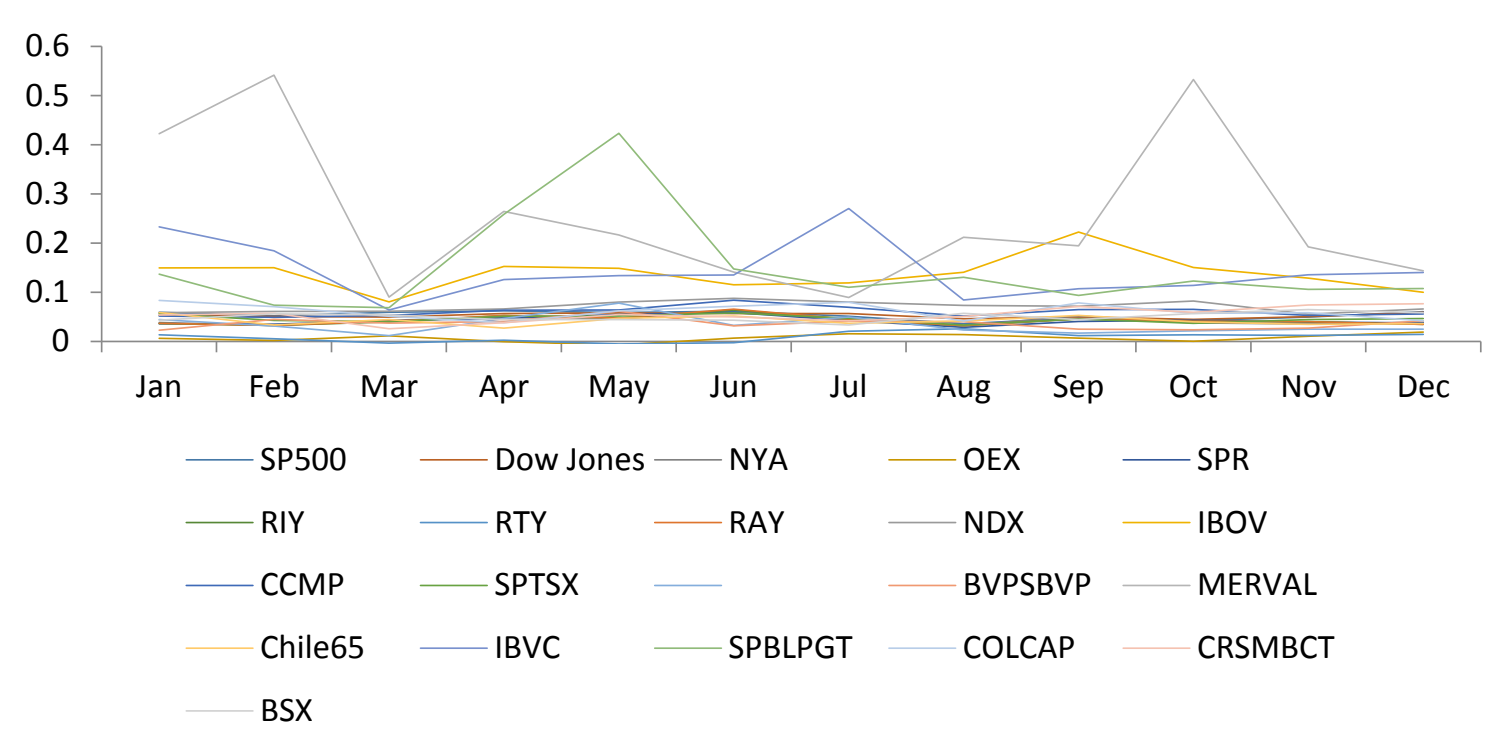

Figure 2. Standard deviation per month - Americas

Data source: Bloomberg 


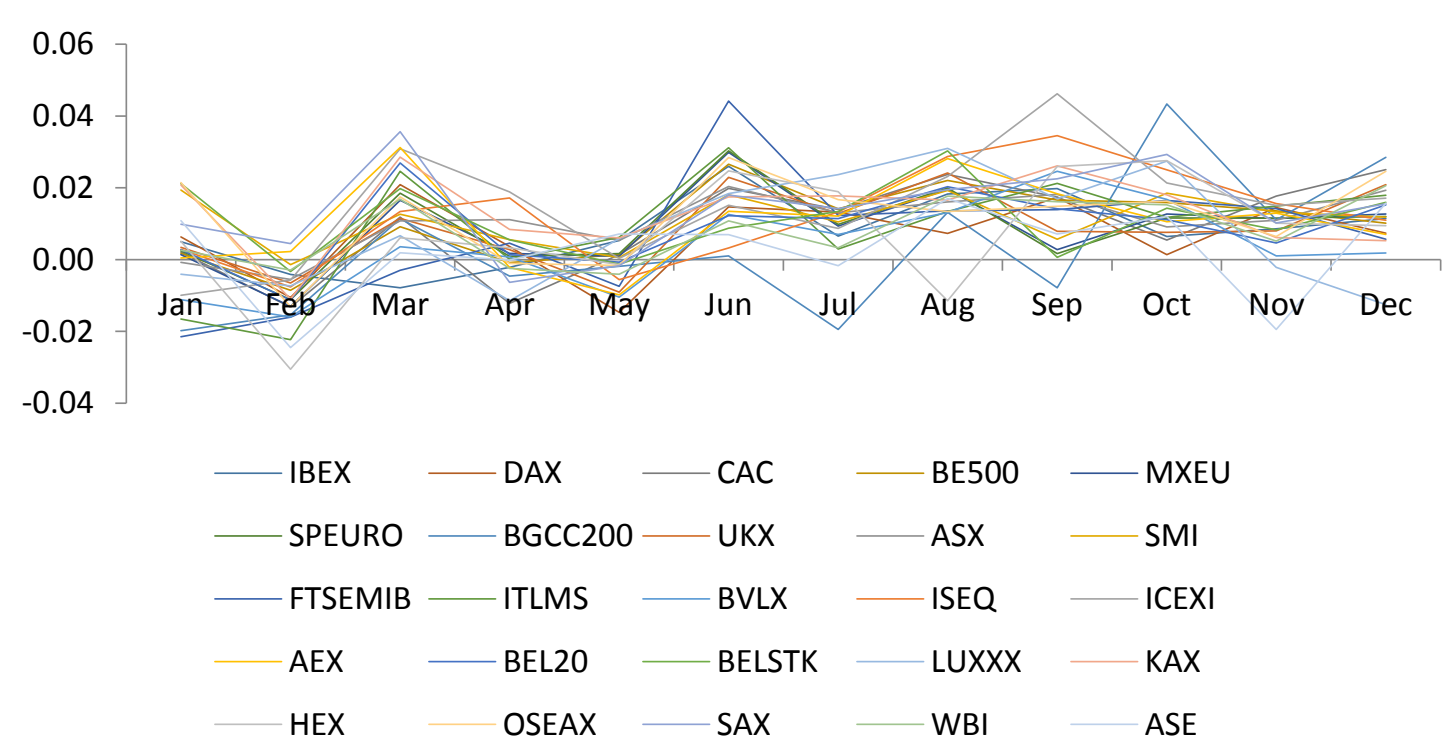

Figure 3. Average return per month - Western Europe

Data source: Bloomberg

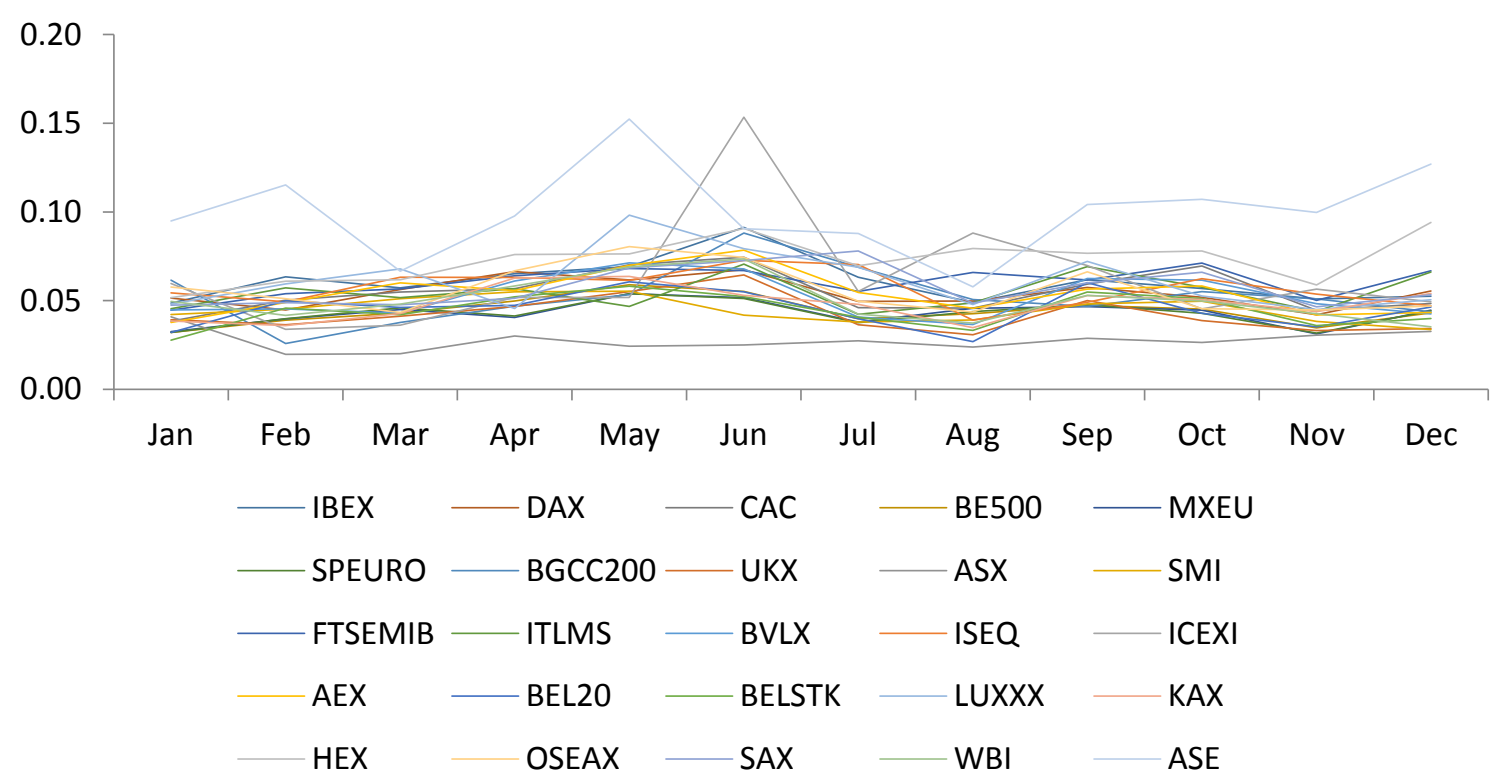

Figure 4. Standard deviation per month - Western Europe

Data source: Bloomberg 


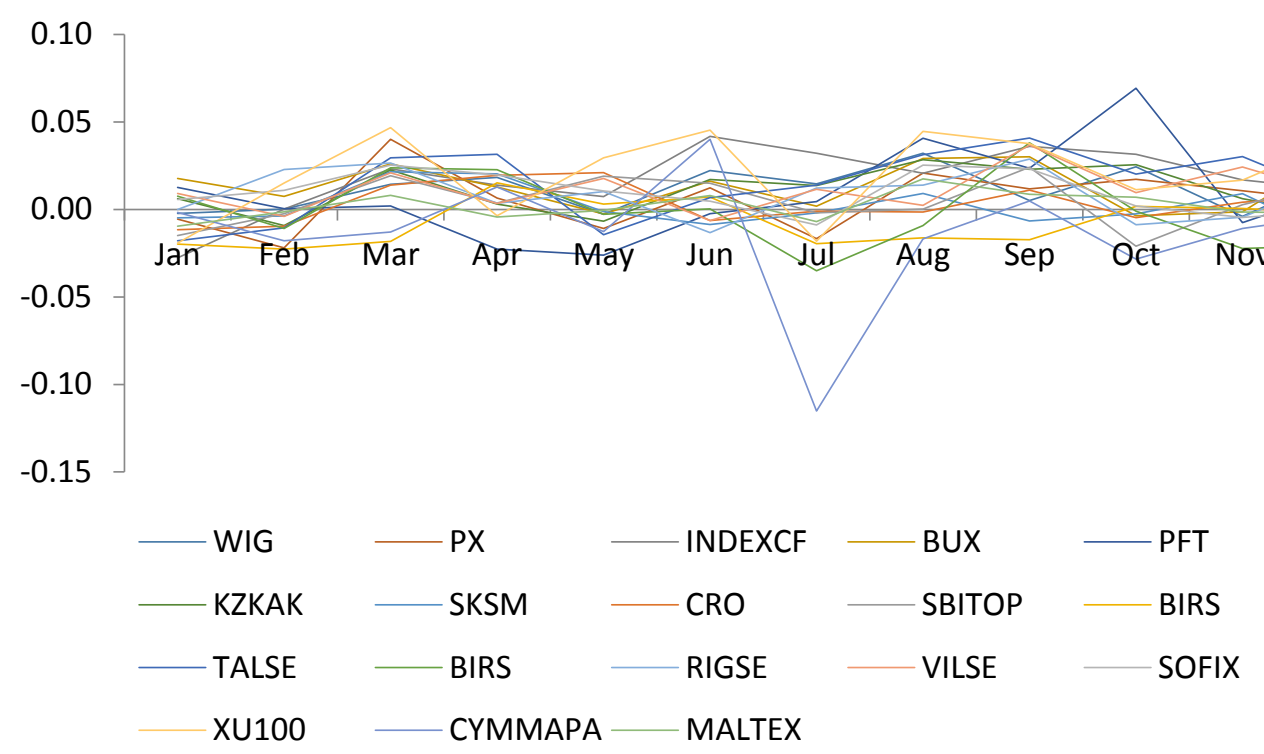

Figure 5. Average return per month - Eastern Europe

Data source: Bloomberg

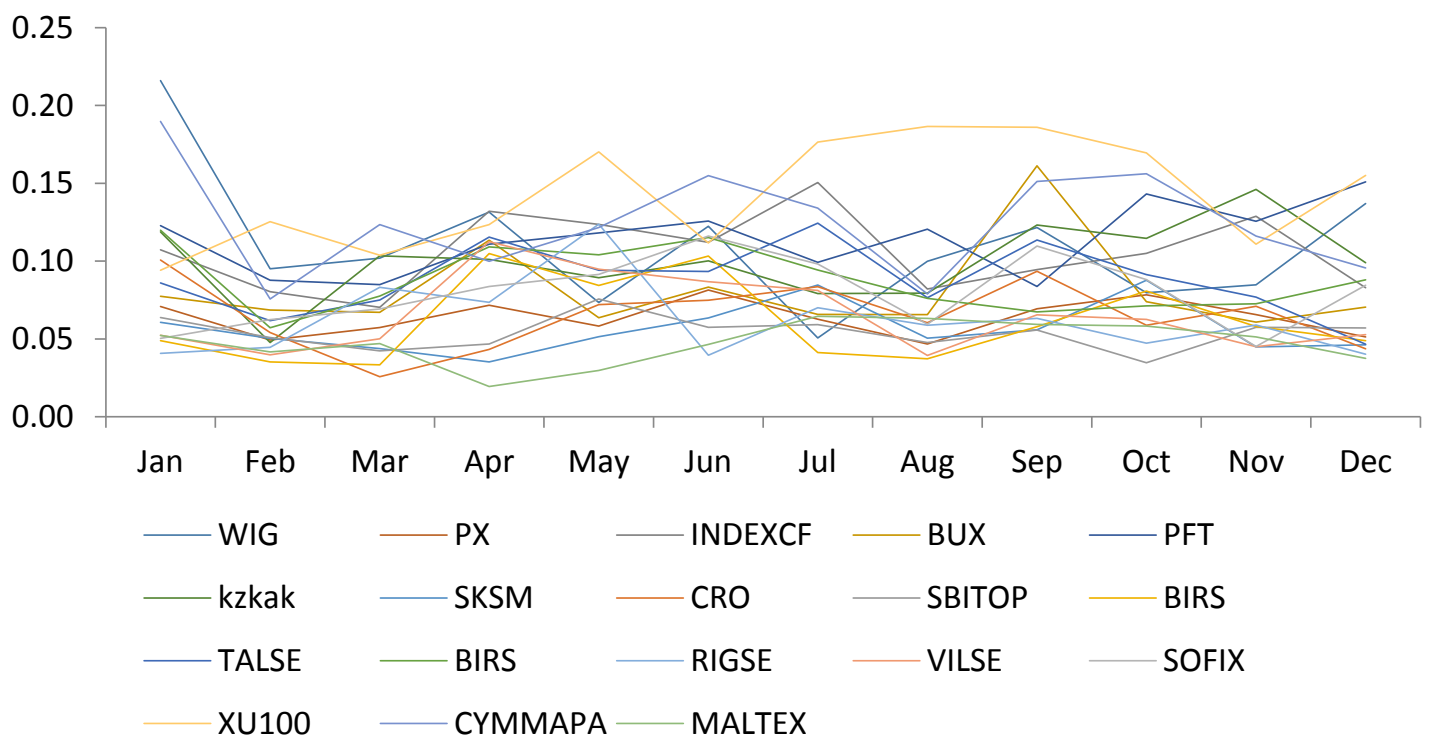

Figure 6. Standard deviation per month - Eastern Europe

Data source: Bloomberg 


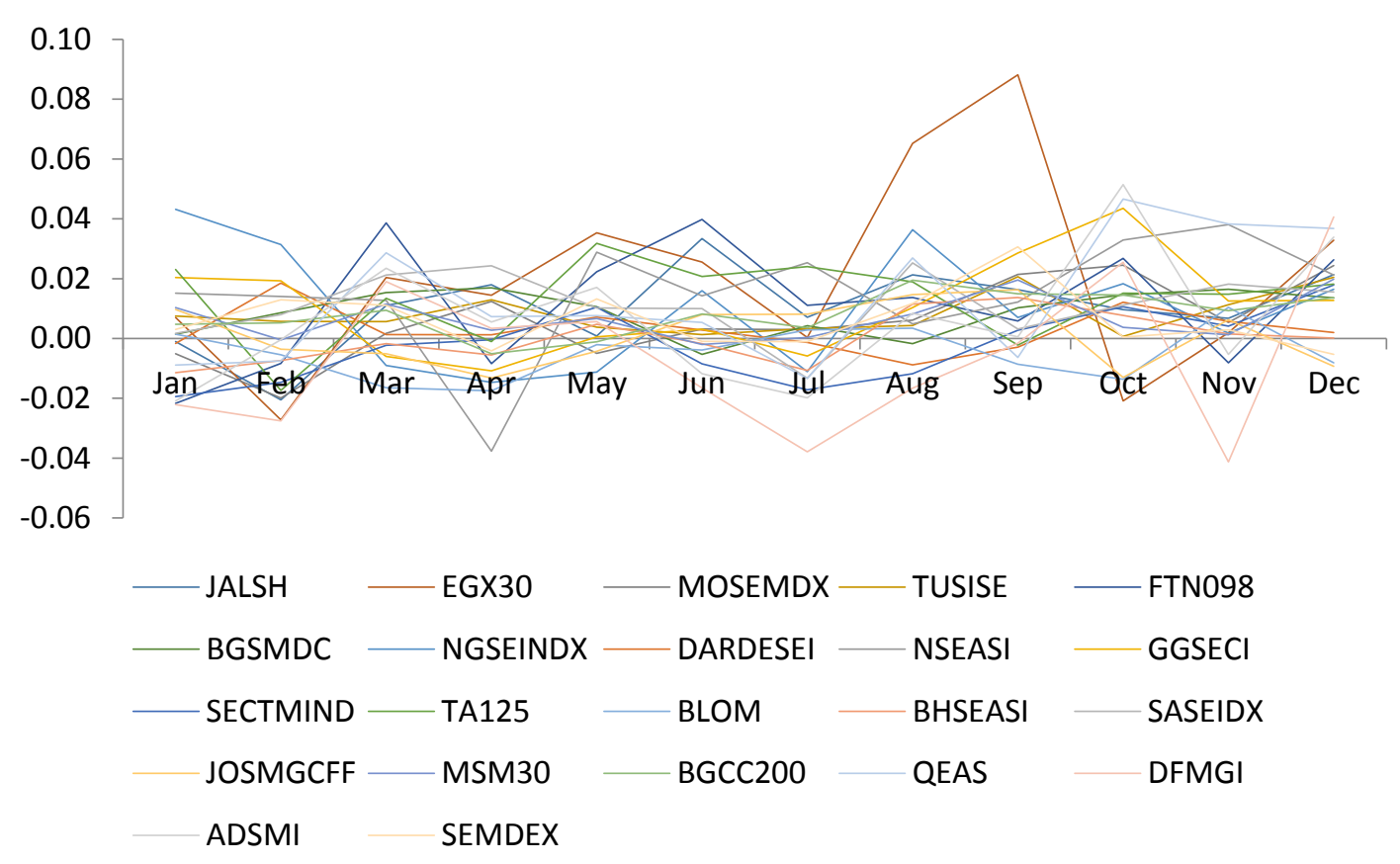

Figure 7. Average return per month - Middle East and Africa

Data source: Bloomberg

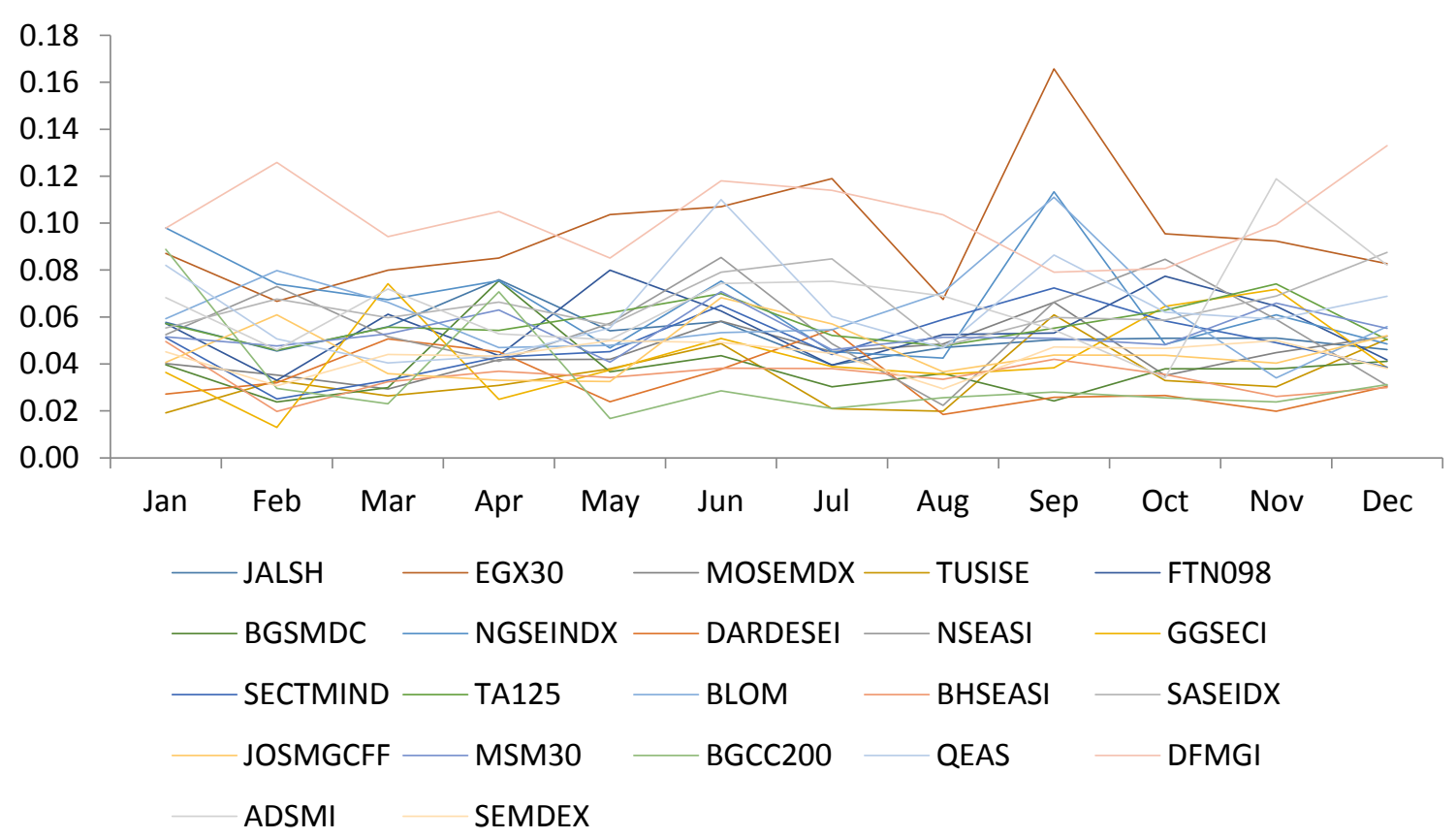

Figure 8. Standard deviation per month - Middle East and Africa

Data source: Bloomberg 


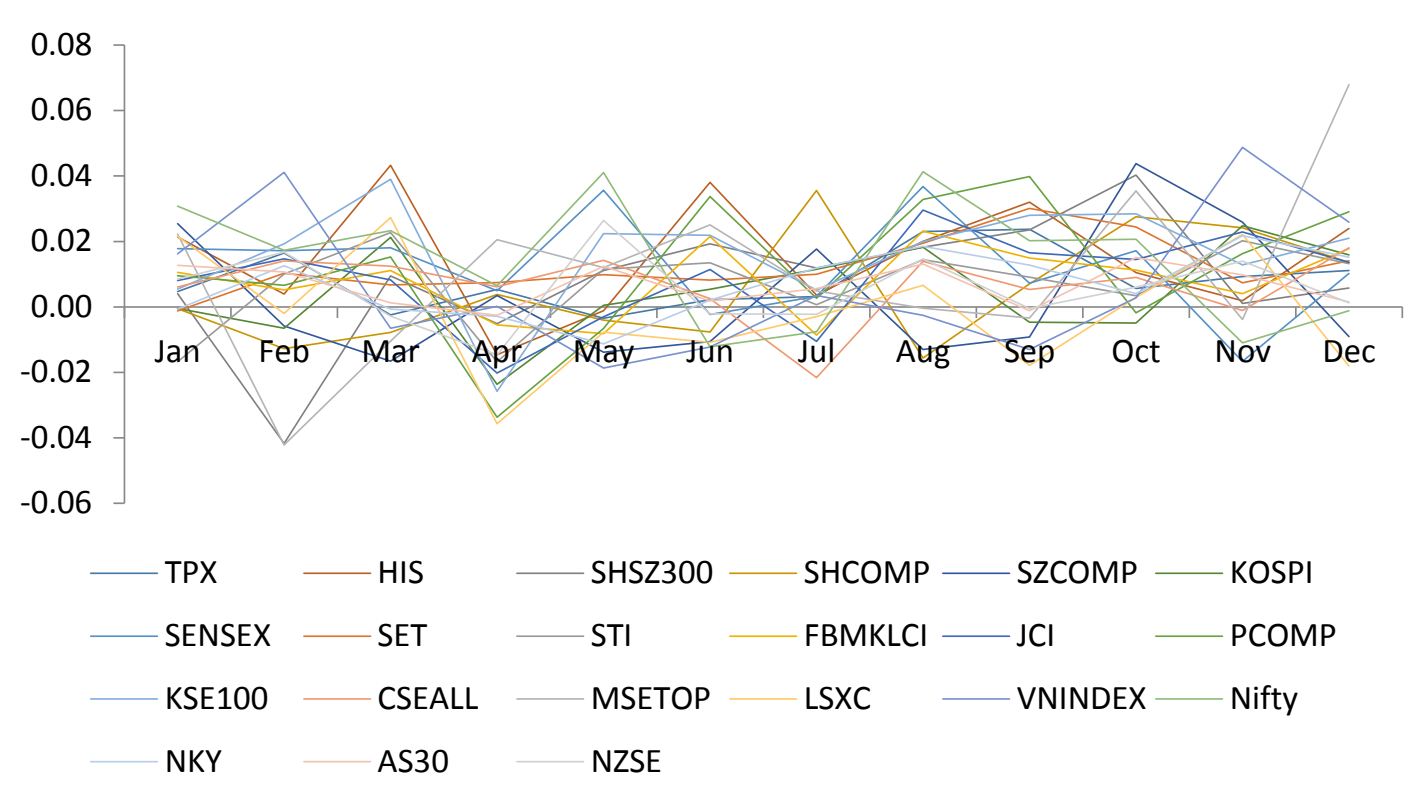

Figure 9. Average return per month - Asia and Oceania

Data source: Bloomberg

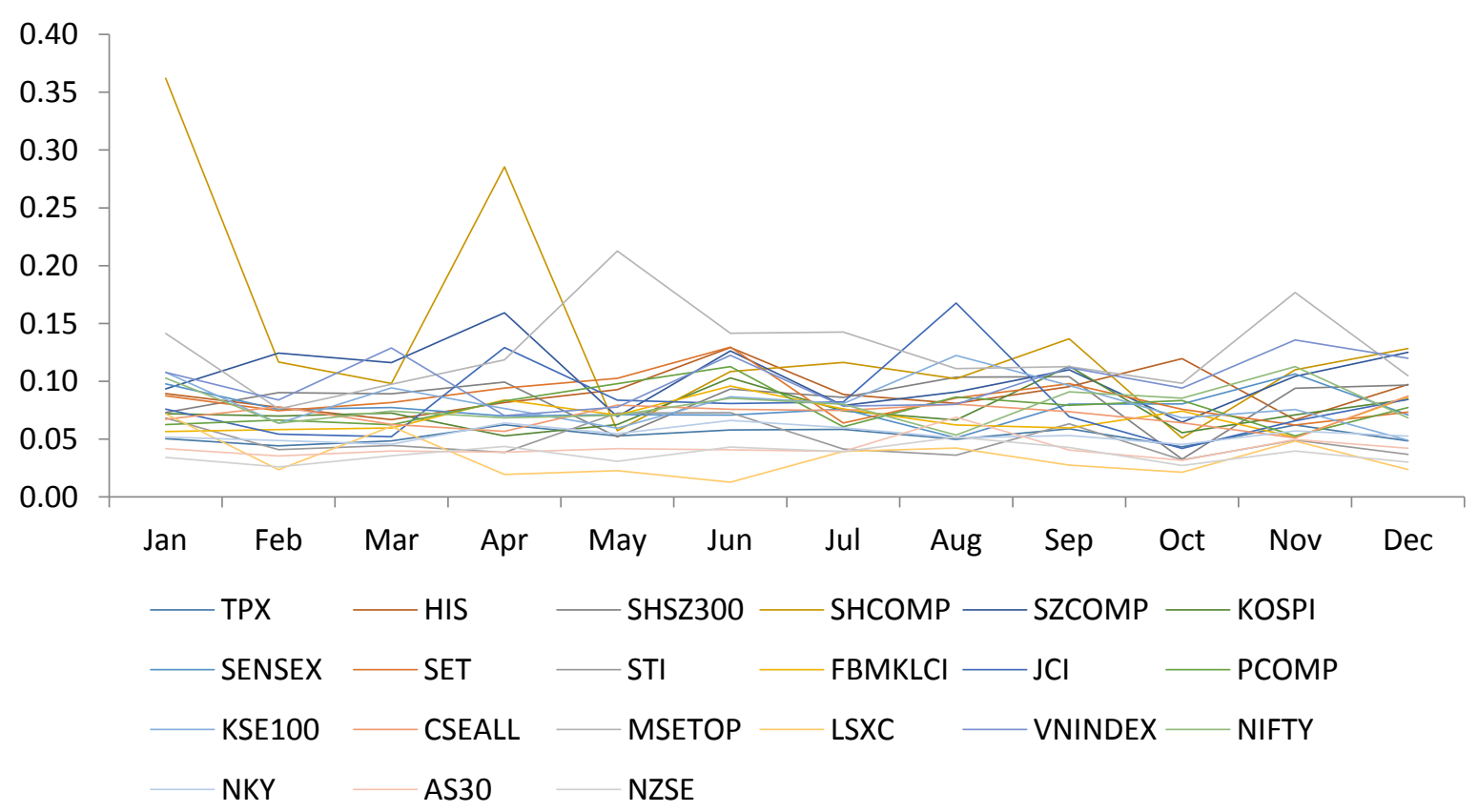

Figure 10. Standard deviation per month - Asia and Oceania

Data source: Bloomberg 
Appendix 2. AD Test

\begin{tabular}{|c|c|c|c|c|c|c|c|c|c|c|c|c|}
\hline Index & Jan & Feb & Mar & Apr & May & Jun & Jul & Aug & Sep & Oct & Nov & Dec \\
\hline S\&P 500 Ind. & 0.001 & 0.001 & 0.002 & 0.001 & 0.001 & 0.001 & 0.112 & 0.007 & 0.271 & 0.039 & 0.001 & 0.001 \\
\hline Dow Jones Industrial & 0.001 & 0.001 & 0.238 & 0.001 & 0.001 & 0.001 & 0.014 & 0.001 & 0.144 & 0.010 & 0.001 & 0.001 \\
\hline Nasdaq Composite Ind. & 0.091 & 0.784 & 0.498 & 0.045 & 0.026 & 0.071 & 0.056 & 0.002 & 0.548 & 0.155 & 0.019 & 0.390 \\
\hline Nasdaq 100 Ind. & 0.103 & 0.064 & 0.197 & 0.052 & 0.080 & 0.025 & 0.238 & 0.001 & 0.291 & 0.115 & 0.075 & 0.472 \\
\hline N.Y. Stock Ex Comp. & 0.343 & 0.327 & 0.346 & 0.161 & 0.063 & 0.001 & 0.033 & 0.072 & 0.672 & 0.944 & 0.372 & 0.702 \\
\hline S\&P 100 Ind. & 0.603 & 0.297 & 0.323 & 0.105 & 0.270 & 0.007 & 0.211 & 0.073 & 0.468 & 0.507 & 0.565 & 0.114 \\
\hline S\&P 1500 Ind. & 0.361 & 0.212 & 0.689 & 0.048 & 0.330 & 0.086 & 0.027 & 0.189 & 0.187 & 0.239 & 0.786 & 0.542 \\
\hline Russell 1000 Ind. & 0.486 & 0.323 & 0.201 & 0.201 & 0.529 & 0.001 & 0.081 & 0.025 & 0.480 & 0.246 & 0.416 & 0.383 \\
\hline Russell 2000 Ind. & 0.197 & 0.429 & 0.588 & 0.030 & 0.170 & 0.004 & 0.104 & 0.545 & 0.869 & 0.975 & 0.028 & 0.516 \\
\hline Russell 3000 Ind. & 0.501 & 0.326 & 0.182 & 0.232 & 0.451 & 0.001 & 0.051 & 0.022 & 0.553 & 0.265 & 0.326 & 0.394 \\
\hline S\&P Toronto Com. Ind. & 0.001 & 0.010 & 0.497 & 0.001 & 0.001 & 0.002 & 0.096 & 0.014 & 0.507 & 0.188 & 0.001 & 0.011 \\
\hline S\&P BMV Mexico Ind. & 0.878 & 0.060 & 0.587 & 0.356 & 0.640 & 0.172 & 0.848 & 0.001 & 0.321 & 0.047 & 0.792 & 0.389 \\
\hline Bolsa de Valores Pan. & 0.001 & 0.010 & 0.497 & 0.001 & 0.001 & 0.002 & 0.096 & 0.014 & 0.507 & 0.188 & 0.001 & 0.011 \\
\hline Buenos Aires St. Ind. & 0.001 & 0.001 & 0.347 & 0.001 & 0.001 & 0.814 & 0.302 & 0.001 & 0.001 & 0.001 & 0.001 & 0.003 \\
\hline Ibovespa Ind. & 0.001 & 0.001 & 0.817 & 0.004 & 0.002 & 0.198 & 0.001 & 0.001 & 0.001 & 0.001 & 0.001 & 0.701 \\
\hline Chile 65 Ind. & 0.002 & 0.248 & 0.923 & 0.028 & 0.361 & 0.886 & 0.021 & 0.064 & 0.322 & 0.929 & 0.220 & 0.856 \\
\hline Caracas Stock Exc. Ind. & 0.001 & 0.001 & 0.143 & 0.076 & 0.049 & 0.006 & 0.001 & 0.032 & 0.161 & 0.049 & 0.003 & 0.009 \\
\hline S\&P BVL Peru Ind. & 0.001 & 0.111 & 0.259 & 0.001 & 0.001 & 0.002 & 0.046 & 0.001 & 0.977 & 0.001 & 0.002 & 0.568 \\
\hline Colombia Colcap Ind. & 0.382 & 0.124 & 0.121 & 0.302 & 0.044 & 0.034 & 0.095 & 0.989 & 0.098 & 0.066 & 0.780 & 0.153 \\
\hline BCT Costa Rica Ind. & 0.008 & 0.006 & 0.013 & 0.928 & 0.061 & 0.663 & 0.012 & 0.001 & 0.055 & 0.084 & 0.001 & 0.001 \\
\hline Bermuda Stock Ex. Ind. & 0.003 & 0.062 & 0.642 & 0.495 & 0.495 & 0.028 & 0.004 & 0.002 & 0.058 & 0.651 & 0.001 & 0.291 \\
\hline Bloomberg Europe 500 & 0.209 & 0.400 & 0.308 & 0.002 & 0.166 & 0.316 & 0.306 & 0.517 & 0.264 & 0.371 & 0.552 & 0.057 \\
\hline MSCI Europe Ind. & 0.307 & 0.836 & 0.229 & 0.004 & 0.060 & 0.228 & 0.167 & 0.705 & 0.429 & 0.439 & 0.561 & 0.040 \\
\hline S\&P Europe 350 Ind. & 0.330 & 0.807 & 0.178 & 0.006 & 0.067 & 0.273 & 0.270 & 0.653 & 0.408 & 0.333 & 0.562 & 0.041 \\
\hline DAX Ind. & 0.782 & 0.274 & 0.271 & 0.001 & 0.009 & 0.530 & 0.022 & 0.057 & 0.475 & 0.487 & 0.723 & 0.063 \\
\hline CAC 40 Ind. & 0.955 & 0.931 & 0.677 & 0.031 & 0.461 & 0.028 & 0.364 & 0.616 & 0.054 & 0.173 & 0.680 & 0.435 \\
\hline Ibex 35 Ind. & 0.038 & 0.332 & 0.638 & 0.130 & 0.326 & 0.016 & 0.397 & 0.962 & 0.247 & 0.145 & 0.423 & 0.359 \\
\hline FTSE 100 Ind. & 0.630 & 0.312 & 0.883 & 0.601 & 0.951 & 0.001 & 0.641 & 0.940 & 0.386 & 0.437 & 0.990 & 0.199 \\
\hline FTSE All Share Ind. & 0.414 & 0.601 & 0.663 & 0.099 & 0.058 & 0.001 & 0.006 & 0.063 & 0.001 & 0.023 & 0.003 & 0.009 \\
\hline Swiss Market Ind. & 0.265 & 0.887 & 0.816 & 0.001 & 0.369 & 0.028 & 0.415 & 0.291 & 0.941 & 0.354 & 0.852 & 0.810 \\
\hline FTSE MIB BorsaItaliana & 0.890 & 0.279 & 0.682 & 0.077 & 0.035 & 0.021 & 0.953 & 0.534 & 0.554 & 0.244 & 0.425 & 0.380 \\
\hline FTSE Italia All & 0.522 & 0.393 & 0.202 & 0.192 & 0.179 & 0.047 & 0.257 & 0.438 & 0.146 & 0.624 & 0.620 & 0.359 \\
\hline PSI All Share Ind. & 0.361 & 0.684 & 0.432 & 0.232 & 0.105 & 0.124 & 0.951 & 0.785 & 0.881 & 0.442 & 0.535 & 0.722 \\
\hline Irish Stock Ex. Overall & 0.689 & 0.155 & 0.761 & 0.053 & 0.067 & 0.001 & 0.051 & 0.007 & 0.524 & 0.542 & 0.592 & 0.068 \\
\hline Iceland Stock Exc. Ind. & 0.085 & 0.599 & 0.001 & 0.260 & 0.017 & 0.001 & 0.070 & 0.001 & 0.192 & 0.031 & 0.028 & 0.081 \\
\hline Amsterdam St. Ex. In. & 0.830 & 0.708 & 0.061 & 0.024 & 0.007 & 0.001 & 0.152 & 0.176 & 0.033 & 0.709 & 0.763 & 0.111 \\
\hline Belgium 20 Ind. & 0.628 & 0.574 & 0.032 & 0.050 & 0.038 & 0.001 & 0.097 & 0.855 & 0.203 & 0.027 & 0.605 & 0.166 \\
\hline Brussels St. Exc. Ind. & 0.306 & 0.334 & 0.016 & 0.014 & 0.022 & 0.017 & 0.037 & 0.666 & 0.543 & 0.122 & 0.702 & 0.054 \\
\hline Luxemburg Sto. Exc. Ind. & 0.321 & 0.923 & 0.928 & 0.496 & 0.009 & 0.001 & 0.663 & 0.334 & 0.528 & 0.032 & 0.070 & 0.026 \\
\hline OMX Copenhagen Ind. & 0.330 & 0.958 & 0.685 & 0.773 & 0.012 & 0.003 & 0.131 & 0.648 & 0.417 & 0.925 & 0.693 & 0.032 \\
\hline OMX Helsinki Ind. & 0.419 & 0.128 & 0.393 & 0.016 & 0.782 & 0.584 & 0.082 & 0.007 & 0.932 & 0.010 & 0.594 & 0.109 \\
\hline Oslo All Share Ind. & 0.557 & 0.591 & 0.666 & 0.021 & 0.103 & 0.081 & 0.396 & 0.393 & 0.215 & 0.425 & 0.243 & 0.452 \\
\hline OMX Stockholm All & 0.121 & 0.575 & 0.194 & 0.107 & 0.207 & 0.028 & 0.510 & 0.311 & 0.577 & 0.067 & 0.290 & 0.258 \\
\hline
\end{tabular}




\begin{tabular}{|c|c|c|c|c|c|c|c|c|c|c|c|c|}
\hline Vienna St. Exc. Ind. & 247 & 0.700 & 0.532 & 0.083 & 0.019 & 0.003 & 0.217 & 0.827 & 0.168 & 0.256 & 0.228 & 0.47 \\
\hline Athens St. Exc. Gen. Ind. & 0.240 & 0.001 & 0.025 & 0.015 & 0.036 & 0.107 & 0.198 & 0.854 & 0.543 & 0.013 & 0.002 & $0 \Omega 0$ \\
\hline Jarsaw St. Exc. Ind. & .001 & .061 & 0.011 & .001 & 0.053 & 0.088 & 0.333 & 0.001 & .002 & .411 & .007 & .06 \\
\hline rague St. Exc. Ind. & .040 & 0.652 & 0.238 & 0.018 & 0.097 & 0.045 & 0.327 & 0.074 & 0.017 & 0.685 & 0.559 & 016 \\
\hline IICEX Ind. & 017 & .164 & 0.047 & 0.011 & 0.706 & 0.048 & & 0.375 & & 0.009 & .002 & .21 \\
\hline Budapest St. Exc. Ind. & .377 & 0.595 & 0.867 & 0.047 & 0.091 & 0.026 & 0.776 & 0.024 & 0.001 & 0.705 & 0.081 & 080 \\
\hline kraine PFTS Ind. & .091 & 0.178 & 0.673 & 0.039 & 0.293 & 0.486 & 0.190 & 0.003 & 0.095 & 0.532 & 0.019 & \\
\hline Kazakhstan St.Ind. & .154 & 0.784 & 0.320 & 0.374 & 0.064 & 0.004 & 0.186 & 0.174 & 0.013 & 0.001 & 0.001 & $000-2$ \\
\hline Slovak Share Ind. & .867 & 0.402 & 0.040 & 0.835 & 0.058 & 0.204 & 0.021 & 0.065 & & 0.001 & 0.562 & 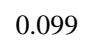 \\
\hline Lagreb St. Exc. Ind. & .001 & 0.605 & 0.941 & 0.848 & 0.752 & 0.001 & 0.025 & 0.001 & 0.833 & 0.070 & 0.316 & 011 \\
\hline jubljana St. Exc. Ind. & 640 & 0.401 & 0.632 & 0.196 & 0.105 & 0.005 & 0.544 & 0.244 & 0.388 & 0.425 & 0.306 & 000 \\
\hline St. Exc. Ind. Rep. Srpska & 324 & 0.077 & 0.803 & 0.004 & 0.027 & 0.015 & 0.883 & 0.072 & 0.003 & 0.087 & 0.090 & 041 \\
\hline OMX Tallinn Ind. & .007 & 0.167 & 0.001 & 0.001 & 0.051 & 0.002 & 0.013 & 0.576 & 0.006 & 0.696 & 0.094 & 0.18 \\
\hline MBI 10 Ind. & 0.001 & 0.203 & 0.097 & 0.058 & 0.036 & 0.050 & 0.474 & 0.166 & 0.990 & 0.048 & 0.001 & 000 \\
\hline OMX Riga Ind. & .307 & 0.625 & 0.001 & 0.015 & 0.008 & 0.371 & 0.181 & 0.150 & & 0.134 & 0.869 & 000 \\
\hline OMX Vilnius Ind. & 0.179 & 0.020 & 0.060 & 0.001 & 0.073 & 0.006 & 0.111 & 0.016 & 0.171 & 0.678 & 0.894 & 0028 \\
\hline Bulgaria St. Exc. Ind. & .535 & 0.560 & 0.370 & 0.024 & 0.181 & 0.013 & 0.044 & 0.009 & 0.137 & 0.003 & 0.107 & 0.00 \\
\hline The BorsaIstambul 100 & 0.204 & 0.022 & 0.738 & 0.193 & 0.017 & 0.844 & 0.039 & 0.001 & 0.001 & 0.052 & 0.546 & 024 \\
\hline Cyprus General Exc. Ind. & .031 & 0.492 & 0.236 & 0.001 & 0.157 & 0.350 & 0.804 & 0.130 & 0.713 & 0.134 & 0.342 & 0.21 \\
\hline Malta St. Exc. Ind. & 0.008 & 0.124 & 0.682 & 0.091 & 0.851 & 0.277 & 0.001 & 0.001 & 0.038 & 0.420 & 0.013 & \\
\hline FTSE JSE Africa All Ind. & .271 & 0.223 & 0.173 & 0.001 & 0.439 & 0.554 & 0.123 & 0.620 & & 0.984 & 0.206 & 0.3 \\
\hline EGX 30 Ind. & 0.964 & 0.688 & 0.861 & 0.531 & 0.813 & 0.003 & 0.023 & 0.397 & 0.934 & 0.401 & 0.044 & 081 \\
\hline MADEX Casablanca I & 111 & 0.009 & 0.961 & 0.282 & 0.559 & 0.042 & 0.990 & 0.044 & 0.213 & 0.814 & 0.706 & 0.73 \\
\hline Tunisia St. Exc. Ind. & 0.957 & 0.518 & 0.200 & 0.334 & 0.610 & 0.456 & 0.642 & 0.886 & 0.287 & 0.371 & 0.111 & 0.42 \\
\hline FTSE JSE Namibia Ind. & .158 & 0.875 & 0.585 & 0.338 & 0.147 & 0.033 & 0.609 & 0.701 & 0.700 & 0.023 & 0.082 & 0.02 \\
\hline Botswana Gaborone Ind. & 0.044 & 0.246 & 0.421 & 0.001 & 0.009 & 0.001 & 0.587 & 0.001 & 0.824 & 0.219 & 0.060 & $00 \Omega$ \\
\hline Nigerian Sto. Exc. Ind. & 575 & 0.628 & 0.458 & 0.569 & 0.847 & 0.701 & 0.539 & 0.488 & 0.162 & 0.076 & 0.183 & $0.2 \mathrm{a}$ \\
\hline Tanzania All Share Ind. & 0.055 & 0.023 & 0.097 & 0.001 & 0.036 & 0.004 & 0.019 & 0.177 & 0.106 & 0.099 & 0.609 & 0.61 \\
\hline Nairobi Sec. Exc. All Ind. & 0.599 & 0.034 & 0.024 & 0.596 & 0.023 & 0.013 & 0.280 & 0.461 & & 0.001 & 0.815 & 0.10 \\
\hline Ghana Composite Ind. & 0.285 & 0.042 & 0.268 & 0.935 & 0.210 & 0.859 & 0.077 & 0.297 & 0.21 & 0.398 & 0.042 & 000 \\
\hline Kuwait St. Exc. Ind. & 0.093 & 0.694 & 0.573 & 0.990 & 0.053 & 0.002 & & & & & 0.749 & 0.1 \\
\hline Tel Aviv St. Exc. Ind. & 213 & 0.107 & 0.690 & 0.495 & 0.057 & 0.005 & 0.478 & 0.977 & 0.220 & 0.008 & 0.721 & 082 \\
\hline Blom Stock Index Ind. & .001 & 0.001 & 0.149 & 0.621 & 0.024 & 0.022 & & & & & 0.943 & 0.06 \\
\hline Bahrain Bourse All Share & .253 & 0.347 & 0.506 & 0.012 & 0.001 & 0.168 & 0.011 & 0.492 & 0.613 & 0.953 & 0.378 & 0.55 \\
\hline Tadawull All Share Ind. & 0.235 & 0.035 & 0.111 & 0.456 & 0.071 & 0.081 & 0.442 & 0.025 & & & 0.946 & $0.12+32+3$ \\
\hline Amman St. Exc. Ind. & 927 & 0.228 & 0.005 & 0.111 & 0.886 & 0.001 & 0.251 & 0.280 & 0.189 & 0.316 & 0.511 & 0.03 \\
\hline Huscat MSM 30 ind. & 809 & 0.219 & 0.010 & 0.043 & 0.343 & 0.001 & 0.090 & 0.063 & & & 0.003 & 000 \\
\hline Bloomberg GCC 200 Ind. & 0.570 & 0.091 & 0.713 & 0.336 & 0.088 & 0.027 & 0.546 & 0.299 & 0.547 & 0.076 & 0.731 & 080 \\
\hline QE All Share & 300 & 0.134 & 0.979 & 0.321 & 0.544 & 0.002 & 0.328 & 0.693 & & 0.080 & 0.901 & 0.21 \\
\hline DFM G. Ind. & 0.147 & 0.004 & 0.139 & 0.051 & 0.242 & 0.036 & 0.049 & 0.756 & 0.675 & 0.736 & 0.214 & 0.26 \\
\hline A ou Dnabi Gen. ind. & 0.234 & 0.038 & 0.191 & 0.558 & 0.683 & 0.006 & 0.022 & 0.382 & 0.847 & 0.215 & 0.001 & 0.00 \\
\hline Mauritius SEMDEX Ind. & 0.001 & 0.180 & 0.164 & 0.003 & 0.255 & 0.003 & 0.001 & 0.897 & 0.451 & 0.004 & 0.007 & 0.12 \\
\hline Tokyo St. Exc. Ind. Ind. & 0.136 & 0.078 & 0.450 & 0.561 & 0.252 & 0.005 & 0.535 & 0.145 & 0.240 & 0.245 & 0.351 & 0.013 \\
\hline Nikkei 225 Ind. & 0.072 & 0.141 & 0.500 & 0.294 & 0.229 & 0.006 & 0.169 & 0.894 & 0.216 & 0.334 & 0.800 & 0.03 \\
\hline NSE Nifty 50 Ind. & 0.118 & 0.120 & 0.054 & 0.781 & 0.288 & 0.380 & 0.800 & 0.254 & 0.651 & 0.012 & 0.038 & $0.8 / 0$ \\
\hline S\&P BSE Sensex Ind. & 0.075 & 0.080 & 0.023 & 0.116 & 0.244 & 0.027 & 0.806 & 0.044 & 0.818 & 0.003 & 0.033 & 0.35 \\
\hline
\end{tabular}




\begin{tabular}{lllllllllllll} 
HIS Index Ind. & 0.410 & 0.020 & 0.302 & 0.215 & 0.272 & 0.001 & 0.001 & 0.001 & 0.485 & 0.001 & 0.191 & 0.001 \\
CSI 300 Ind. & 0.741 & 0.677 & 0.077 & 0.285 & 0.044 & 0.080 & 0.941 & 0.103 & 0.805 & 0.833 & 0.487 & 0.083 \\
Shanghai Comp. Ind. & 0.001 & 0.035 & 0.299 & 0.001 & 0.777 & 0.102 & 0.018 & 0.133 & 0.004 & 0.246 & 0.131 & 0.043 \\
Shenzhen Comp. Ind. & 0.717 & 0.206 & 0.119 & 0.001 & 0.824 & 0.023 & 0.896 & 0.490 & 0.880 & 0.525 & 0.968 & 0.005 \\
Korea St. Exc. Kospi Ind. & 0.551 & 0.011 & 0.162 & 0.493 & 0.783 & 0.008 & 0.186 & 0.193 & 0.001 & 0.728 & 0.088 & 0.740 \\
Bangkok SET Ind. & 0.232 & 0.384 & 0.141 & 0.039 & 0.726 & 0.001 & 0.253 & 0.165 & 0.256 & 0.257 & 0.610 & 0.001 \\
Straits Time Ind. & 0.023 & 0.173 & 0.690 & 0.616 & 0.127 & 0.001 & 0.808 & 0.473 & 0.682 & 0.034 & 0.003 & 0.026 \\
FTSE Bursa KLCI Ind. & 0.307 & 0.045 & 0.054 & 0.012 & 0.194 & 0.002 & 0.007 & 0.001 & 0.510 & 0.004 & 0.722 & 0.157 \\
Jakarta Stock Exc. Ind. & 0.745 & 0.519 & 0.404 & 0.001 & 0.251 & 0.015 & 0.015 & 0.001 & 0.217 & 0.024 & 0.445 & 0.323 \\
Philippine St. Exc. Ind. & 0.154 & 0.028 & 0.248 & 0.035 & 0.069 & 0.075 & 0.831 & 0.001 & 0.744 & 0.002 & 0.926 & 0.297 \\
Karachi KSE100 Ind. & 0.154 & 0.028 & 0.248 & 0.035 & 0.069 & 0.075 & 0.831 & 0.001 & 0.744 & 0.002 & 0.926 & 0.297 \\
Sri Lanka Colombo Ind. & 0.048 & 0.178 & 0.065 & 0.408 & 0.075 & 0.119 & 0.016 & 0.003 & 0.199 & 0.140 & 0.068 & 0.015 \\
MSE top 20 Ind. & 0.081 & 0.793 & 0.024 & 0.424 & 0.001 & 0.122 & 0.015 & 0.785 & 0.377 & 0.577 & 0.001 & 0.341 \\
Laos Composite & 0.027 & 0.088 & 0.420 & 0.905 & 0.570 & 0.562 & 0.614 & 0.283 & 0.990 & 0.068 & 0.140 & 0.804 \\
Ho Chi Minh St. Ind. & 0.055 & 0.641 & 0.150 & 0.018 & 0.224 & 0.039 & 0.634 & 0.001 & 0.024 & 0.099 & 0.228 & 0.240 \\
Australian All Ord. Ind. & 0.097 & 0.061 & 0.001 & 0.051 & 0.261 & 0.022 & 0.021 & 0.001 & 0.164 & 0.458 & 0.013 & 0.001 \\
S\&P NZX All Ind. & 0.056 & 0.703 & 0.586 & 0.088 & 0.639 & 0.122 & 0.115 & 0.653 & 0.084 & 0.331 & 0.329 & 0.058 \\
\hline
\end{tabular}

Data source: Bloomberg

Appendix 3. Lillie Test

\begin{tabular}{|c|c|c|c|c|c|c|c|c|c|c|c|c|}
\hline Index & Jan & Feb & Mar & Apr & May & Jun & Jul & Aug & Sep & Oct & Nov & Dec \\
\hline S\&P 500 Ind. & 0.010 & 0.017 & 0.065 & 0.001 & 0.006 & 0.001 & 0.045 & 0.014 & 0.429 & 0.044 & 0.001 & 0.001 \\
\hline Dow Jones Industrial Ind. & 0.001 & 0.019 & 0.500 & 0.002 & 0.001 & 0.001 & 0.011 & 0.001 & 0.061 & 0.066 & 0.027 & 0.001 \\
\hline Nasdaq Composite Ind. & 0.012 & 0.500 & 0.500 & 0.153 & 0.017 & 0.140 & 0.102 & 0.023 & 0.500 & 0.441 & 0.016 & 0.381 \\
\hline Nasdaq 100 Ind. & 0.028 & 0.121 & 0.500 & 0.297 & 0.170 & 0.073 & 0.176 & 0.001 & 0.216 & 0.227 & 0.165 & 0.336 \\
\hline N.Y. Stock Ex Comp. Ind. & 0.092 & 0.500 & 0.164 & 0.335 & 0.159 & 0.006 & 0.190 & 0.126 & 0.500 & 0.500 & 0.338 & 0.500 \\
\hline S\&P 100 Ind. & 0.500 & 0.179 & 0.232 & 0.108 & 0.196 & 0.018 & 0.223 & 0.041 & 0.500 & 0.500 & 0.193 & 0.143 \\
\hline S\&P 1500 Ind. & 0.149 & 0.194 & 0.423 & 0.082 & 0.500 & 0.174 & 0.146 & 0.233 & 0.073 & 0.353 & 0.406 & 0.224 \\
\hline Russell 1000 Ind. & 0.252 & 0.500 & 0.136 & 0.214 & 0.500 & 0.007 & 0.316 & 0.037 & 0.263 & 0.294 & 0.500 & 0.302 \\
\hline Russell 2000 Ind. & 0.194 & 0.498 & 0.500 & 0.008 & 0.141 & 0.091 & 0.060 & 0.452 & 0.500 & 0.500 & 0.063 & 0.243 \\
\hline Russell 3000 Ind. & 0.148 & 0.449 & 0.090 & 0.196 & 0.494 & 0.005 & 0.207 & 0.030 & 0.298 & 0.428 & 0.500 & 0.242 \\
\hline S\&P Toronto Com. Ind. & 0.003 & 0.012 & 0.500 & 0.016 & 0.019 & 0.017 & 0.186 & 0.027 & 0.309 & 0.075 & 0.014 & 0.017 \\
\hline S\&P BMV Mexico Ind. & 0.500 & 0.296 & 0.324 & 0.142 & 0.500 & 0.317 & 0.500 & 0.003 & 0.449 & 0.258 & 0.500 & 0.500 \\
\hline Bolsa de Valores Pan. Ind. & 0.003 & 0.012 & 0.500 & 0.016 & 0.019 & 0.017 & 0.186 & 0.027 & 0.309 & 0.075 & 0.014 & 0.017 \\
\hline Buenos Aires St. Ex. Ind. & 0.001 & 0.001 & 0.077 & 0.001 & 0.008 & 0.500 & 0.500 & 0.002 & 0.078 & 0.001 & 0.001 & 0.018 \\
\hline IbovespaInd. & 0.003 & 0.001 & 0.500 & 0.048 & 0.010 & 0.246 & 0.061 & 0.003 & 0.003 & 0.001 & 0.012 & 0.500 \\
\hline Chile 65 Ind. & 0.015 & 0.391 & 0.500 & 0.082 & 0.292 & 0.500 & 0.007 & 0.181 & 0.112 & 0.500 & 0.203 & 0.500 \\
\hline Caracas Stock Exc. Ind. & 0.007 & 0.001 & 0.065 & 0.193 & 0.054 & 0.032 & 0.001 & 0.048 & 0.307 & 0.075 & 0.032 & 0.035 \\
\hline S\&P BVL Peru Ind. & 0.001 & 0.027 & 0.254 & 0.001 & 0.001 & 0.007 & 0.145 & 0.001 & 0.500 & 0.002 & 0.030 & 0.500 \\
\hline Colombia ColcapInd. & 0.286 & 0.103 & 0.220 & 0.098 & 0.102 & 0.271 & 0.401 & 0.500 & 0.055 & 0.096 & 0.500 & 0.330 \\
\hline BCT Costa Rica Ind. & 0.044 & 0.003 & 0.004 & 0.500 & 0.094 & 0.298 & 0.023 & 0.010 & 0.112 & 0.305 & 0.004 & 0.002 \\
\hline Bermuda Stock Ex. Ind. & 0.013 & 0.263 & 0.500 & 0.500 & 0.500 & 0.056 & 0.013 & 0.007 & 0.020 & 0.372 & 0.020 & 0.372 \\
\hline Bloomberg Europe 500 & 0.464 & 0.235 & 0.280 & 0.002 & 0.179 & 0.392 & 0.146 & 0.458 & 0.089 & 0.500 & 0.500 & 0.036 \\
\hline MSCI Europe Ind. & 0.253 & 0.500 & 0.434 & 0.005 & 0.094 & 0.500 & 0.072 & 0.500 & 0.358 & 0.500 & 0.500 & 0.011 \\
\hline S\&P Europe 350 Ind. & 0.365 & 0.500 & 0.360 & 0.006 & 0.099 & 0.500 & 0.065 & 0.500 & 0.202 & 0.471 & 0.500 & 0.015 \\
\hline
\end{tabular}




\begin{tabular}{|c|c|c|c|c|c|c|c|c|c|c|c|c|}
\hline Ouctobe Dorec DA & 0.437 & 0.233 & 0.475 & 0.006 & 0.189 & 0.500 & 0.159 & 0.304 & 0.500 & 0.500 & 0.391 & \\
\hline AC 40 Ind. & 0.500 & 0.500 & 0.500 & 0.019 & 0.117 & 0.015 & 0.430 & 0.500 & 0.183 & 0.500 & 0.500 & \\
\hline bex 35 Ind. & 200 & 0.378 & 0.422 & 0.174 & 0.227 & 0.059 & 0.500 & 0.500 & 0.420 & 0.095 & 0.405 & 0.46 \\
\hline TSE 100 Ind. & 500 & 363 & 500 & 500 & 500 & 001 & .500 & .500 & 500 & 464 & 500 & \\
\hline TSE All Share Ind. & 0.500 & 0.500 & 0.500 & .035 & 0.111 & 0.007 & 0.029 & 0.232 & 0.001 & 0.184 & 0.012 & \\
\hline wiss Market Ind. & 500 & .500 & .500 & 013 & 380 & 017 & 0.500 & .373 & 0.500 & .325 & 500 & \\
\hline FTSE MIB BorsaItaliana & 0.500 & 0.295 & 0.500 & 0.034 & 0.249 & 0.012 & 0.500 & 0.500 & 0.410 & 0.223 & 0.303 & \\
\hline TSE Italia All Share Ind. & .317 & 0.365 & 0.120 & 0.115 & 0.500 & 0.028 & 0.143 & 0.500 & 0.031 & 0.411 & 0.500 & \\
\hline SI All Share Ind. & 0.333 & 0.500 & 0.171 & 0.212 & 0.076 & 0.213 & 0.500 & 0.500 & 0.500 & 0.500 & 0.368 & \\
\hline ish Stock Ex. Overall Ind. & 0.500 & 0.144 & 0.500 & 0.083 & & 0.004 & 0.161 & 0.014 & 0.417 & 0.500 & & \\
\hline eland Stock Exc. Ind. & 0.344 & 0.500 & 0.001 & 0.223 & 0.158 & 0.001 & 0.334 & 0.001 & 0.340 & 0.122 & 0.085 & \\
\hline msterdam Stock Ex. Ind. & 0.500 & 0.500 & 0.082 & 0.199 & 0.035 & 0.007 & 0.465 & 0.182 & 0.172 & 0.500 & 0.500 & \\
\hline elgium 20 Ind. & 0.379 & 0.500 & 0.039 & 0.042 & 0.104 & 0.033 & 0.474 & 0.500 & 0.385 & 0.422 & 0.334 & \\
\hline russels St. Exc. Ind. & 0.244 & 0.291 & 0.093 & 0.004 & 0.164 & 0.079 & 0.076 & 0.500 & 0.500 & 0.221 & 0.500 & \\
\hline uxemburg Sto. Exc. Ind. & 0.278 & 0.500 & 0.500 & 0.486 & 0.031 & 0.025 & 0.500 & 0.429 & 0.500 & 0.159 & 0.262 & \\
\hline MX Copenhagen Ind. & 0.500 & 0.500 & 0.500 & 0.431 & 0.034 & 0.074 & 0.160 & 0.500 & 0.428 & 0.500 & 0.305 & \\
\hline MX Helsinki Ind. & 0.236 & 0.073 & 0.249 & 0.032 & 0.500 & 0.500 & 0.036 & 0.101 & 0.343 & 0.088 & 0.500 & \\
\hline slo All Share Ind. & 0.500 & 0.494 & 0.500 & 0.072 & 0.328 & 0.071 & 0.296 & 0.146 & 0.315 & 0.500 & 0.198 & \\
\hline MY Stockholm All & 0.090 & 0.500 & 0.116 & 0.062 & 0.263 & 0.016 & 0.500 & 0.500 & 0.500 & 0.092 & 0.325 & \\
\hline ienna St. Exc. Ind, & 0.265 & 0.500 & 0.500 & 0.108 & 0.195 & 0.119 & 0.211 & 0.500 & 0.115 & 0.500 & 0.240 & \\
\hline Athens St. Exc. Gen. Ind. & 0.252 & 0.011 & 0.026 & 0.084 & 0.084 & 0.063 & 0.393 & 0.500 & 0.500 & 0.044 & 0.066 & \\
\hline Varsaw St. Exc. Ind. & 0.001 & 0.408 & 0.109 & 0.001 & 0.180 & 0.042 & 0.125 & 0.011 & 0.036 & 0.423 & 0.003 & \\
\hline rague St. Exc. Ind. & 0.105 & 0.500 & 0.128 & 0.060 & 0.195 & 0.100 & 0.384 & 0.130 & 0.018 & 0.493 & 0.261 & \\
\hline IICEX Ind. & 0.003 & 0.229 & 0.018 & 0.034 & 0.500 & 0.070 & 0.041 & 0.500 & 0.385 & 0.049 & 0.030 & \\
\hline udapest St. Exc. Ind. & 0.500 & 0.441 & 0.500 & 0.152 & 0.191 & 0.219 & 0.500 & 0.041 & 0.001 & 0.483 & 0.044 & \\
\hline Jkraine PFTS Ind. & 0.389 & 0.067 & 0.500 & 0.057 & 0.413 & 0.473 & 0.048 & 0.031 & 0.142 & 0.500 & 0.115 & \\
\hline azakbstan St Fxc Ind & 0.163 & 0.500 & 0.497 & 0.208 & 0.339 & 0.011 & 0.311 & 0.401 & 0.006 & 0.001 & 0.022 & \\
\hline lovak Share & 0.500 & 0.500 & 0.018 & 0.313 & 0.069 & 0.080 & 0.014 & 0.157 & 0.156 & 0.003 & 0.246 & \\
\hline agreb St. Exc. Ind. & 0.006 & 0.239 & 0.500 & 0.500 & 0.467 & 0.001 & 0.095 & 0.001 & 0.500 & 0.320 & 0.297 & \\
\hline jubljana St. Exc. Ind. & 0.500 & 0.346 & 0.500 & 0.241 & & 0.024 & 0.500 & 0.384 & & & & \\
\hline t. Exc. Ind. Rep. Srpska & 0.500 & 0.036 & 0.500 & 0.019 & 0.068 & 0.008 & 0.500 & 0.056 & 0.013 & 0.124 & 0.045 & \\
\hline 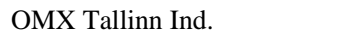 & 0.012 & 0.113 & 0.001 & 0.001 & 0.059 & & 0.008 & 0.427 & & & & \\
\hline PI 10 Ind & 0.001 & 0.254 & 0.095 & 0.183 & 0.039 & 0.040 & 0.439 & 0.264 & 0.500 & 0.146 & 0.003 & \\
\hline 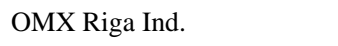 & 0.467 & 0.500 & 0.020 & 0.005 & 0.010 & 0.471 & 0.500 & & & & & \\
\hline MX Vilnius Ind. & 0.125 & 0.036 & 0.047 & 0.001 & 0.029 & 0.035 & 0.059 & 0.033 & 0.500 & 0.500 & 0.500 & \\
\hline Ganta st. EX. MU. & 0.326 & 0.500 & 0.500 & 0.026 & 0.265 & 0.068 & 0.025 & 0.006 & & & & \\
\hline The BorsaIstambul 100 & 0.134 & 0.090 & 0.500 & 0.319 & 0.008 & 0.500 & 0.051 & 0.001 & 0.001 & 0.012 & 0.500 & \\
\hline yprus General Exc. mal. & 0.074 & 0.342 & 0.098 & 0.005 & 0.132 & 0.255 & 0.500 & 0.216 & 0.500 & & & \\
\hline Malta St. Exc. Ind. & 0.059 & 0.206 & 0.500 & 0.018 & 0.500 & 0.182 & 0.014 & 0.017 & 0.073 & 0.210 & 0.001 & \\
\hline TSE JSE Africa All Ind. & 0.124 & 0.324 & 0.031 & 0.002 & 0.335 & 0.500 & 0.058 & 0.500 & 0.404 & 0.500 & 0.329 & \\
\hline Egyptian Exc. EGX 30 Ind. & 0.500 & 0.500 & 0.500 & 0.446 & 0.500 & 0.015 & 0.044 & 0.301 & 0.500 & 0.204 & 0.086 & \\
\hline MADEX CasablancaInd. & 0.266 & 0.015 & 0.500 & 0.479 & 0.500 & 0.101 & 0.500 & 0.061 & 0.302 & 0.500 & 0.500 & \\
\hline Tunisia St. Exc. Ind. & 0.500 & 0.078 & 0.500 & 0.052 & 0.500 & 0.217 & 0.406 & 0.500 & 0.242 & 0.500 & 0.285 & \\
\hline TSE JSE Namibia Ind. & 0.189 & 0.500 & 0.500 & 0.133 & 0.446 & 0.168 & 0.381 & 0.500 & 0.500 & 0.050 & 0.016 & \\
\hline otswana Gaborone Ind. & 0.037 & 0.309 & 0.278 & 0.001 & 0.032 & 0.006 & 0.500 & 0.012 & 0.500 & 0.500 & 0.085 & \\
\hline & & & & & & & & & & & & \\
\hline
\end{tabular}




\begin{tabular}{|c|c|c|c|c|c|c|c|c|c|c|c|c|}
\hline Tanzania All Share Ind. & 0.022 & 0.041 & 0.085 & 0.001 & 0.017 & 0.022 & 0.022 & 0.063 & 0.165 & 0.058 & 0.500 & 0.500 \\
\hline Nairobi Sec. Exc. All Ind. & 0.273 & 0.007 & 0.008 & 0.500 & 0.075 & 0.161 & 0.500 & 0.500 & 0.500 & 0.006 & 0.500 & 0.266 \\
\hline Ghana Composite Ind. & 0.500 & 0.189 & 0.366 & 0.500 & 0.156 & 0.500 & 0.140 & 0.123 & 0.170 & 0.500 & 0.037 & 0.169 \\
\hline Kuwait St. Exc. Ind. & 0.154 & 0.431 & 0.447 & 0.500 & 0.019 & 0.007 & 0.416 & 0.027 & 0.500 & 0.204 & 0.500 & 0.053 \\
\hline Tel Aviv St. Exc. Ind. & 0.240 & 0.166 & 0.373 & 0.500 & 0.037 & 0.079 & 0.345 & 0.500 & 0.500 & 0.019 & 0.500 & 0.500 \\
\hline Blom Stock Index Ind. & 0.001 & 0.001 & 0.178 & 0.500 & 0.102 & 0.021 & 0.289 & 0.001 & 0.001 & 0.003 & 0.500 & 0.289 \\
\hline Bahrain Bourse All Share & 0.345 & 0.398 & 0.190 & 0.055 & 0.001 & 0.171 & 0.006 & 0.500 & 0.500 & 0.500 & 0.389 & 0.356 \\
\hline Tadawull All Share Ind. & 0.339 & 0.104 & 0.320 & 0.500 & 0.274 & 0.360 & 0.437 & 0.114 & 0.175 & 0.500 & 0.500 & 0.259 \\
\hline Amman St. Exc. Ind. & 0.500 & 0.229 & 0.009 & 0.260 & 0.500 & 0.006 & 0.358 & 0.403 & 0.273 & 0.355 & 0.500 & 0.114 \\
\hline Muscat MSM 30 Ind. & 0.500 & 0.254 & 0.015 & 0.121 & 0.500 & 0.062 & 0.373 & 0.315 & 0.248 & 0.165 & 0.001 & 0.247 \\
\hline Bloomberg GCC 200 Ind. & 0.318 & 0.212 & 0.500 & 0.500 & 0.052 & 0.091 & 0.241 & 0.182 & 0.500 & 0.101 & 0.500 & 0.500 \\
\hline QE All Share Ind. & 0.257 & 0.277 & 0.500 & 0.380 & 0.239 & 0.001 & 0.500 & 0.500 & 0.383 & 0.236 & 0.500 & 0.292 \\
\hline Dubai Fin. Mar. Gen. Ind. & 0.369 & 0.032 & 0.100 & 0.062 & 0.300 & 0.049 & 0.095 & 0.453 & 0.500 & 0.500 & 0.100 & 0.255 \\
\hline Abu Dhabi General Ind. & 0.180 & 0.039 & 0.500 & 0.500 & 0.482 & 0.045 & 0.044 & 0.500 & 0.500 & 0.187 & 0.001 & 0.240 \\
\hline Mauritius SEMDEX Ind. & 0.001 & 0.443 & 0.286 & 0.024 & 0.301 & 0.006 & 0.005 & 0.500 & 0.291 & 0.034 & 0.120 & 0.045 \\
\hline Tokyo St. Exc. Ind. Ind. & 0.161 & 0.311 & 0.235 & 0.466 & 0.500 & 0.037 & 0.500 & 0.323 & 0.286 & 0.445 & 0.500 & 0.172 \\
\hline Nikkei 225 Ind. & 0.006 & 0.148 & 0.500 & 0.500 & 0.277 & 0.034 & 0.310 & 0.500 & 0.203 & 0.496 & 0.500 & 0.038 \\
\hline NSE Nifty 50 Ind. & 0.365 & 0.200 & 0.267 & 0.500 & 0.284 & 0.451 & 0.500 & 0.387 & 0.500 & 0.014 & 0.109 & 0.500 \\
\hline S\&P BSE Sensex Ind. & 0.239 & 0.230 & 0.088 & 0.093 & 0.279 & 0.002 & 0.500 & 0.077 & 0.500 & 0.019 & 0.141 & 0.255 \\
\hline HIS Index Ind. & 0.500 & 0.109 & 0.042 & 0.139 & 0.500 & 0.001 & 0.002 & 0.001 & 0.448 & 0.001 & 0.144 & 0.003 \\
\hline CSI 300 Ind. & 0.500 & 0.500 & 0.007 & 0.334 & 0.021 & 0.088 & 0.500 & 0.247 & 0.500 & 0.500 & 0.305 & 0.212 \\
\hline Shanghai Comp. Ind. & 0.001 & 0.005 & 0.149 & 0.001 & 0.500 & 0.050 & 0.033 & 0.081 & 0.036 & 0.234 & 0.046 & 0.162 \\
\hline Shenzhen Comp. Ind. & 0.500 & 0.249 & 0.162 & 0.012 & 0.500 & 0.014 & 0.500 & 0.500 & 0.500 & 0.500 & 0.500 & 0.038 \\
\hline Korea St. Exc. KospiInd. & 0.500 & 0.100 & 0.077 & 0.500 & 0.500 & 0.010 & 0.017 & 0.248 & 0.005 & 0.500 & 0.086 & 0.500 \\
\hline Bangkok SET Ind. & 0.024 & 0.490 & 0.492 & 0.039 & 0.500 & 0.006 & 0.038 & 0.228 & 0.386 & 0.500 & 0.500 & 0.001 \\
\hline Straits Time Ind. & 0.053 & 0.500 & 0.500 & 0.500 & 0.089 & 0.011 & 0.500 & 0.269 & 0.500 & 0.058 & 0.029 & 0.276 \\
\hline FTSE Bursa KLCI Ind. & 0.253 & 0.078 & 0.045 & 0.178 & 0.351 & 0.018 & 0.027 & 0.005 & 0.463 & 0.008 & 0.500 & 0.361 \\
\hline Jakarta Stock Exc. Ind. & 0.261 & 0.500 & 0.216 & 0.004 & 0.254 & 0.031 & 0.058 & 0.001 & 0.075 & 0.076 & 0.500 & 0.150 \\
\hline Philippine St. Exc. Ind. & 0.261 & 0.003 & 0.460 & 0.179 & 0.336 & 0.185 & 0.500 & 0.006 & 0.500 & 0.011 & 0.500 & 0.325 \\
\hline Karachi KSE100 Ind. & 0.261 & 0.003 & 0.460 & 0.179 & 0.336 & 0.185 & 0.500 & 0.006 & 0.500 & 0.011 & 0.500 & 0.325 \\
\hline Sri Lanka Colombo Ind. & 0.033 & 0.318 & 0.117 & 0.500 & 0.154 & 0.155 & 0.011 & 0.009 & 0.100 & 0.254 & 0.154 & 0.069 \\
\hline MSE top 20 Ind. & 0.010 & 0.500 & 0.094 & 0.311 & 0.001 & 0.215 & 0.080 & 0.500 & 0.500 & 0.356 & 0.018 & 0.500 \\
\hline Laos Securities Composite & 0.040 & 0.054 & 0.469 & 0.500 & 0.500 & 0.342 & 0.500 & 0.282 & 0.500 & 0.031 & 0.070 & 0.500 \\
\hline Ho Chi Minh St. Ind. & 0.052 & 0.500 & 0.073 & 0.109 & 0.500 & 0.133 & 0.500 & 0.001 & 0.016 & 0.267 & 0.346 & 0.436 \\
\hline Australian All Ord. Ind. & 0.296 & 0.270 & 0.010 & 0.047 & 0.500 & 0.041 & 0.011 & 0.001 & 0.500 & 0.324 & 0.074 & 0.001 \\
\hline S\&P NZX All Ind. & 0.123 & 0.422 & 0.443 & 0.131 & 0.500 & 0.329 & 0.419 & 0.273 & 0.069 & 0.169 & 0.165 & 0.049 \\
\hline
\end{tabular}

Data source: Bloomberg 